\title{
An Analysis of ANNNI Model by Peierl's Contour Method
}

\author{
E. I. Dinaburg and Ya. G. Sinai \\ Landau Institute of Theoretical Physics, Academy of Sciences, Moscow, USSR
}

\begin{abstract}
For the three-dimensional ANNNI model a converging expression for the curve of the coexistence of the (3.3)-phase and ferromagnetic phase is derived for low temperatures using a new extension of the Peierls contour method.
\end{abstract}

\section{Description of ANNNI Model and Formulation of the Result}

We consider a classical spin model on the lattice $\mathbb{Z}^{3}$, where the spin variables take the values \pm 1 and the Hamiltonian has the form

$$
\begin{aligned}
H(\varphi(U))= & -J_{0} \sum_{\left(x, x^{\prime}\right) \in U_{\text {hor }}} \varphi(x) \varphi\left(x^{\prime}\right)-J_{1} \sum_{\left(x, x^{\prime}\right) \in U_{\text {ver }}^{(1)}} \varphi(x) \varphi\left(x^{\prime}\right) \\
& +J_{2} \sum_{\left(x, x^{\prime}\right) \in U_{\text {ver }}^{(2)}} \varphi(x) \varphi\left(x^{\prime}\right) .
\end{aligned}
$$

Here $U \subset \mathbb{Z}^{(3)}$ is a finite set, $U_{\text {hor }}$ is the set of horizontal bonds, $U_{\text {ver }}^{(1)}\left(U_{\text {ver }}^{(2)}\right)$ is the set of vertical bonds of length $1(2)$; in all cases the ends belong to $U, \varphi(x)$ is the spin variable at the point $x, \varphi(U)$ is the notation for a configuration on $U$. Parameters $J_{0}, J_{1}, J_{2}$ are positive coupling constants.

This model is called the axial next-nearest neighbor Ising model or, briefly, ANNNI model. It was introduced more than twenty years ago by Domb [1] and Elliott [2], and recently attracted much attention in connection with experimental results concerning compounds of rare-earth elements (see [3]).

This paper was motivated by the deep analysis of the phase diagram of the ANNNI model performed in the paper by Fisher and Selke (see [4]). Using a formal perturbation theory the authors have shown that for low temperatures $T$ there are infinitely many separation-phase lines on the plane $\left(T, J_{1} / J_{2}\right)$. All these lines start in the point $(0,1 / 2)$, where an infinite degeneracy of ground states takes place. From the mathematical point of view these lines determine the values of parameters where the number of periodic extreme limit Gibbs states is discontinuous.

The main idea of [4] which apparently is of more general importance is that for finite temperatures there appears a splitting of ground states if we take into account one-point spin flips of the least energy. 
We reproduce below the corresponding arguments. Assume that $U$ is a cube of size $l$ and we consider configurations $\varphi(U)$ with periodic boundary conditions. It is easy to see that it is sufficient to look for ground states for which $\varphi(x)$, $x=\left(x_{1}, x_{2}, x_{3}\right)$ depend only on $x_{3}$. All such configurations decompose onto series of horizontal planes $x_{3}=$ const, where $\varphi(x)$ is a constant. We denote by $N_{r}$ $=N_{r}(\varphi(U))$ the number of such series of the width $r$. Assuming that $\sum_{r \geqq 1} N_{r}>1$ we can rewrite (1) as follows:

$$
\begin{aligned}
H(\varphi(U))= & -2 J_{0} l^{3}+l^{3}\left[J_{1} \sum_{r \geqq 1} N_{r}-J_{1} \sum_{r \geqq 1}(r-1) N_{r}\right. \\
& \left.+J_{2} N_{1}+J_{2} \sum_{r \geqq 2}(r-2) N_{r}-2 J_{2} \sum_{r \geqq 2} N_{r}\right] \\
= & -2 J_{0} l^{3}-\left(J_{1}-J_{2}\right) l^{3}+2\left(J_{1}-2 J_{2}\right)\left(\sum_{r \geqq 1} N_{r}-1\right) l^{2}+4 J_{2} N_{1} l^{2} .
\end{aligned}
$$

The first two terms give the energy of the ferromagnetic configuration for which $\varphi(x)=$ const, $x \in U$. For this configuration $N_{l}=1, N_{r}=0$ for $r<l$.

For $J_{1}-2 J_{2}=0$ we have an infinite degeneracy of ground states because all configurations with $N_{1}=0$ are ground states. If $J_{1}-2 J_{2}>0$ then the ground states (g.s.) are ferromagnetic configurations ( $f$-g.s.). If $J_{1}-2 J_{2}<0$ then the ground states are four configurations for which $N_{r}=0$ for $r \neq 2$.

Assume that an inverse temperature $\beta$ is chosen and fixed. In the usual picture of phase transitions of the first kind the limit Gibbs states corresponding to physical phases are concentrated on configurations which look like small islands of perturbations of an underlying "ground state sea." If this picture is valid then the main contribution to the free energy of the corresponding phase comes from onepoint perturbations of the ground state. We shall list now all possible one-point

\begin{tabular}{|c|c|c|c|}
\hline \multicolumn{2}{|c|}{ Configuration } & \multirow{2}{*}{$\begin{array}{l}\begin{array}{l}\text { Perturbed } \\
\text { configuration }\end{array} \\
\begin{array}{l}++--- \\
--+++\end{array}\end{array}$} & \multirow{2}{*}{$\begin{array}{l}\text { Change of energy } \\
\varepsilon_{0}=8 J_{0}\end{array}$} \\
\hline 1) & $\begin{array}{l}+++-- \\
---++\end{array}$ & & \\
\hline 2) & $\begin{array}{l}+++++ \\
-----\end{array}$ & $\begin{array}{l}++-++ \\
--+--\end{array}$ & $\varepsilon_{1}=8 J_{0}+4 J_{1}-4 J_{2}$ \\
\hline 3) & $\begin{array}{l}-++-- \\
--++- \\
+--++ \\
++--+\end{array}$ & $\begin{array}{l}-+--- \\
---+- \\
+-+++ \\
+++--\end{array}$ & $\varepsilon_{2}=8 J_{0}+4 J_{2}$ \\
\hline 4) & $\begin{array}{l}++++- \\
-++++ \\
----+ \\
+----\end{array}$ & $\begin{array}{l}++-+- \\
-+-++ \\
--+-- \\
+-+--\end{array}$ & $\varepsilon_{3}=8 J_{0}+4 J_{1}$ \\
\hline 5) & $\begin{array}{l}-+++- \\
+---+\end{array}$ & $\begin{array}{l}-+-+- \\
+-+-+\end{array}$ & $\varepsilon_{4}=8 J_{0}+4 J_{1}+8 J_{2}$ \\
\hline
\end{tabular}
perturbations and their energies in the case of ANNNI-model. In Table 1 the

Table 1 
configurations which are drawn horizontally are indeed vertical. Also we assume that $J_{0}$ is sufficiently large comparing with $J_{1}, J_{2}$. Otherwise one should take into account two-point perturbations (we are indebted to G. Uimin for this remark).

The table shows that the least energy $\varepsilon_{0}$ have perturbations on the boundaries between different series. Let us denote by $U_{i}$ the set of points where spin flips of type $i$ can happen, $i=0,1,2,3,4$. It is easy to check that $\left|U_{0}\right|=2 l^{3} \sum_{r \geqq 3} N_{r},\left|U_{1}\right|$ $=l^{2} \sum_{r \geqq 5}(r-4) N_{r},\left|U_{3}\right|=2 l^{2} \sum_{r \geqq 4} N_{r},\left|U_{2}\right|=l^{2} N_{2},\left|U_{4}\right|=l^{2} N_{3}$.

Here and further the absolute value of a set means its cardinality. The number of configurations of $k$ non-interacting spin-flips can be written up to terms of the next order as $C_{\left|U_{i}\right|}^{k}$. Using binomial coefficients we neglect an interaction of spin flips. Let us introduce for a ground state $\bar{\varphi}(U)$ the partition function

$$
\Xi^{(a)}(\bar{\varphi}(U))=\sum_{\varphi(U)} \exp \{-\beta H(\varphi(U))\},
$$

where the sum is taken over only those $\varphi(U)$ which arise after some number of noninteracting spin flips from $\bar{\varphi}(U)$. As was said before, in the main order

$$
\begin{aligned}
\Xi^{(a)}(\bar{\varphi}(U)) & \approx \exp \{-\beta H(\bar{\varphi}(U))\} \cdot \prod_{i=0}^{4} \sum_{k=0}^{\left|U_{i}\right|} C_{\left|U_{i}\right|}^{k} e^{-\beta \varepsilon_{i} k} \\
& =\exp \left\{-\beta H(\bar{\varphi}(U))+\sum_{i=0}^{4}\left|U_{i}\right| \ln \left(1+\exp \left(-\beta \varepsilon_{i}\right)\right)\right\} .
\end{aligned}
$$

Thus the approximate value of the free energy corresponding to the ground state $\bar{\varphi}(U)$ is equal to

$$
\begin{aligned}
\ln \Xi^{(a)}(\bar{\varphi}(U))= & \left(2 J_{0}+J_{1}-J_{2}\right) \beta l^{3}-2\left(J_{1}-2 J_{2}\right) \beta \sum_{r \geqq 2} N_{r} \cdot l^{2} \\
& +2 l^{2} e^{-\beta \varepsilon_{0}} \sum_{r \geqq 3} N_{r}+l^{2} e^{-\beta \varepsilon_{1}} \sum_{r \geqq 4}(r-4) N_{r} \\
& +2 l^{2} e^{-\beta \varepsilon_{3}} \sum_{r \geqq 4} N_{r}+l^{2} e^{-\beta \varepsilon_{2}} N_{2}+l^{2} e^{-\beta \varepsilon_{4}} N_{3} .
\end{aligned}
$$

The difference $\varepsilon_{1}-\varepsilon_{2}=4\left(J_{1}-2 J_{2}\right)=4 \delta$. In the domain $\delta=J_{1}-2 J_{2}>0$ we consider the sum

$$
\begin{aligned}
& -2 \beta \delta l^{2} \sum_{r \geqq 2} N_{r}+2 \exp \left\{-\beta \varepsilon_{0}\right\} l^{2} \sum_{r \geqq 3} N_{r} \\
& +\exp \left\{-\beta \varepsilon_{1}\right\} \cdot l^{2} \cdot \sum_{r \geqq 4} N_{r}(r-4)+l^{2} N_{2} e^{-\beta \varepsilon_{2}} \\
\approx & -2 \beta \delta N_{2} l^{2}+\left(-2 \beta \delta+2 \exp \left\{-\beta \varepsilon_{0}\right\}\right) l^{2} \\
& \cdot \sum_{r \geqq 3} N_{r}+\exp \left\{-\beta \varepsilon_{1}\right\} \cdot l^{2} \sum_{r \geqq 4}(r-4) N_{r}+\exp \left\{-\beta \varepsilon_{1}\right\} N_{2} l^{2} \\
\approx & -\left(2 \beta \delta+\exp \left\{-\beta \varepsilon_{1}\right\}\right) l^{2} N_{2}+\left[2\left(-\beta \delta+\exp \left\{-\beta \varepsilon_{0}\right\}\right)\right. \\
& \left.-4 \exp \left\{-\beta \varepsilon_{1}\right\}\right] l^{2} \cdot \sum_{r \geqq 4} N_{r}+\left[2\left(-\beta \delta+\exp \left\{-\beta \varepsilon_{0}\right\}\right)\right. \\
& \left.-3 \exp \left\{-\beta \varepsilon_{1}\right\}\right] N_{3} l^{2}+\exp \left\{-\beta \varepsilon_{1}\right\} l^{3} .
\end{aligned}
$$


If

$$
\delta>0, \quad 2\left(-\beta \delta+\exp \left\{-\beta \varepsilon_{0}\right\}\right)-3 \exp \left\{-\beta \varepsilon_{1}\right\}>0,
$$

then the sum takes the largest value for the ground state for which $N_{2}=N_{r}=0$ for $r \geqq 4$. This ground state consists of series of three horizontal planes of the same sign $((3,3)$-g.s. $)$ If

$$
\delta>0, \quad 2\left(-\beta \delta+\exp \left\{-\beta \varepsilon_{0}\right\}\right)-3 \exp \left\{-\beta \varepsilon_{1}\right\}<0
$$
then the sum takes the largest value when $\sum_{r \geqq 4} N_{r}$ takes the least value, i.e. for $f$-g.s.
If

$$
2\left(-\beta \delta+\exp \left\{-\beta \varepsilon_{0}\right\}\right)-3 \exp \left\{-\beta \varepsilon_{1}\right\}=0,
$$

then

$$
\left[2\left(-\beta \delta+\exp \left\{-\beta \varepsilon_{0}\right\}\right)-4 \exp \left\{-\beta \varepsilon_{1}\right\}\right]<0
$$

and the sum takes the largest value for $N_{2}=N_{r}=0, r \geqq 4$. Thus if we neglect terms of smallness less than $\exp \left\{-\beta \varepsilon_{1}\right\}$ then for

$$
\delta_{0}=\beta^{-1} \exp \left\{-\beta \varepsilon_{0}\right\}-3 / 2 \beta^{-1} \exp \left\{-\beta \varepsilon_{1}\right\}
$$

the free energies of $f$-g.s. and (3,3)-g.s. coincide. In other words the equality (2) gives an approximate equation for the co-existence of ferromagnetic and (3,3)-phases.

The arguments presented above give evidence for the following picture: there exist $\beta_{0}$ and a curve $\delta=\delta(\beta)$ defined for $\beta>\beta_{0}$ and close to $\delta_{0}(\beta)$ up to terms of order less than $\exp \left\{-\beta \varepsilon_{1}\right\}$ such that for all $\beta>\beta_{0}$ and $\delta>\delta(\beta)$ there are two limit Gibbs states which are small perturbations of f.g.s. (see [6]), for $\delta<\delta(\beta)$ and close enough to $\delta_{0}(\beta)$ there are six limit Gibbs states which are small perturbations of six $(3,3)$-g.s. while for $\delta=\delta(\beta)$ we have eight limit Gibbs states corresponding to the coexistence of f.g.s. and (3,3)-g.s.

Now we can describe the main result of this paper. We develop a version of Peierls contour method suitable for the ANNNI model. We shall see that it has several peculiarities comparing with the usual situation (see [6]). With the help of the new technique we show the following theorem.

Main Theorem. For large enough $\beta$ there exists a continuous function $\delta=\delta(\beta)$ such that for $J_{1}-2 J_{2}=2 \delta(\beta)$ the ANNNI model has eight periodic limit Gibbs states.

One can already see from the previous discussion a special role of isolated spin flips with the energies $\varepsilon_{0}, \varepsilon_{1}$ and $\varepsilon_{2}$. All functions of $\beta$ which tend to zero faster than $\beta^{-1} \exp \left\{-\beta \varepsilon_{1}\right\}$ will be called very small (v.s.). In particular, we shall see that $\delta(\beta)$ $-\delta_{0}(\beta)$ is v.s. Also we put

$$
d(x, y)=\max \left(\left|x_{1}-y_{1}\right|+\left|x_{2}-y_{2}\right|,\left|x_{3}-y_{3}\right|\right)
$$

for $x, y \in \mathbb{Z}^{3}$.

\section{Boundaries for ANNNI Model}

Assume that a configuration $\varphi$ is given which coincides outside a finite set either with a f.g.s. or with a (3,3)-g.s. We shall say that $\varphi$ at a point $x=\left(x_{1}, x_{2}, x_{3}\right) \in \mathbb{Z}^{3}$ is 
in a ferromagnetic phase (f.ph) if $\varphi(y)$ coincides with a f.g.s. for all $y, d(x, y) \leqq 9$. Also $\varphi$ is in (3,3)-phase ((3,3)-ph) at $x \in \mathbb{Z}^{3}$ if there is a (3,3)-g.s. $\psi$ and an interval $(a, b)$, $b-a=18$ such that 1) $\psi\left(x_{1}, x_{2}, a-1\right) \neq \psi\left(x_{1}, x_{2}, a\right), \psi\left(x_{1}, x_{2}, b-1\right) \neq \psi\left(x_{1}, x_{2}, b\right)$; 2) $a+6 \leqq x_{3}<a+12$; 3) $\varphi(y)$ coincides with $\psi(y)$ for all $y=\left(y_{1}, y_{2}, y_{3}\right)$ such that $a \leqq y_{3}<b,\left|y_{1}-x_{1}\right|+\left|y_{2}-x_{2}\right| \leqq 9$.

Due to this definition any boundary of the domain occupied by the $(3,3)$-phase lies between series of different signs.

All points where $\varphi$ is not in a phase are called boundary points. The set of all boundary points is the boundary of $\varphi$ and will be denoted by $B(\varphi)$.

$\mathrm{Q}(\mathrm{x}), \mathrm{x} \in \mathbb{Z}^{3}$ is the closed unit cube with the centrum at $x$. A set of cubes is called connected if for any two cubes one can find a chain of cubes belonging to the set such that the first one and the last one coincide with the given cubes and every two neighboring cubes of the chain have a non-empty intersection.

We shall identify $B(\varphi)$ with the set of cubes $Q(x), x \in B(\varphi)$. The boundary $B(\varphi)$ can be decomposed onto connected components $B_{1}, B_{2}, \ldots, B_{r}$. The boundary $\partial B_{s}$ of $B_{s}, 1 \leqq s \leqq r$, consists of faces which separate points of $B_{s}$ and $\mathbb{Z}^{3} \backslash B_{s}$. A set of faces is called connected if for any two faces of the set one can find a chain of faces belonging to the set such that the first one and the last one coincide with the given faces and every two neighboring faces of the chain have a non-empty intersection. Each $\partial B_{s}$ is decomposed onto connected components one of which is the exteriour component $\partial B_{s}(\mathrm{ext})$ while the others are interiour components $\partial B_{s k}(\mathrm{int}), 1 \leqq k$ $\leqq K_{s}$. We denote $O_{s k}$ the intrinsic connected components of cubes $Q(x), x \in O_{s k}$, not belonging to $B_{s}$, whose boundaries are exactly $\partial B_{s k}($ int $)$. For each $\partial B_{s}($ ext $), \partial B_{s k}($ int $)$ there is a uniquely defined phase which is adjacent to the component.

A face is called vertical (horizontal) if it is parallel (orthogonal) to the axis $x_{3}$. Each component $\partial B_{s}(\mathrm{ext}), \partial B_{s k}$ (int) has several components of vertical faces $\partial B_{s m}^{(\mathrm{ver})}(\mathrm{ext}), m=1, \ldots, M_{s}, \partial B_{s k m}^{(\mathrm{ver})}(\mathrm{int}), \mathrm{m}=1,2, \ldots, M_{s k}$ and several components of horizontal faces $\partial B_{s n}^{\text {(hor) }}(\mathrm{ext}), n=1, \ldots, N_{s}, \partial B_{s k n}^{\text {(hor) }}(\mathrm{int}), n=1, \ldots, N_{s k}$. Due to our definition of points in the $(3,3)$-phase the horizontal components which separate the boundary from a $(3,3)$-g.s. cut an adjacent $(3,3)$-g.s. just exactly between $( \pm)$ series.

The least component $b^{0}$ of the boundary is the component which appears as a result of an isolated spin-flip inside the sea of a g.s. We write $b^{0}(x)$ if the spin-flip takes place at the point $x$.

Let us take a connected component $B_{p}$ of the boundary $B(\varphi)$ for a configuration $\varphi$, and $\partial B_{p j}^{(\mathrm{ver})}$ be one of components $\partial B_{p m}^{(\mathrm{ver})}(\mathrm{ext}), 1 \leqq m \leqq M_{p}$ or $\partial B_{p k m}^{(\mathrm{ver})}(\mathrm{int}), 1 \leqq m \leqq M_{p k}$. A component $\partial B_{p j}^{(\mathrm{ver})}$ is called small if there is a point $x$ such that $\partial B_{p j}^{\text {(ver) }} C \bar{\partial}\left(b^{0}(x)\right)^{(\text {ver })}$.

A small component $\partial B_{p j}^{(\mathrm{ver})}$ is called filled (empty) if there exists (does not exist) at least one point $y \in \mathbb{Z}^{3}$ such that $Q(y) \subset b^{0}(x) \cap B_{p}$. It is clear that we can make one or several spin-flips after which the small component $\partial B_{p j}^{(\mathrm{ver})}$ disappears while other vertical components do not change. Certainly some components of the horizontal boundary also may change.

A connected component $B_{q}$ is called smooth if none of the components of its vertical boundary is small. By definition any $b^{0}(x)$ is also a smooth component. Having a non-smooth component $B_{q}$ we can make all spin-flips, described above, and get a new configuration $\varphi^{\prime}$ with one or several smooth components appearing from $B_{q}$. 


\section{Contours and Contour Models for ANNNI Model}

Let $\varphi$ coincide at infinity either with a f.g.s. or with a $(3,3)$-g.s. and $B(\varphi)$ be its boundary with the components $B_{i}(\varphi), 1 \leqq i \leqq r$. Two components $B_{i_{1}}(\varphi), B_{i_{2}}(\varphi)$ of $B(\varphi)$ are called $s$-connected if the distance between $\left(\partial B_{i_{1}}\right)^{(\text {(hor) }}$ and $\left(\partial B_{i_{2}}\right)^{(\text {hor })}$ is not more than 38 . This notion gives a possibility to decompose $B(\varphi)$ onto maximal components which we shall call s-components and denote by $b_{1}, b_{2}, \ldots, b_{p}, p \leqq r$. The components $B_{i}(\varphi) \subset b_{j}$ are called connected components belonging to the $s$-component $b_{j}$. For each $b_{j}$ a function $p h$ is defined on $\partial b_{j}$ whose value on a face is a ground state which is adjacent to the face.

Definition 1. A contour $\gamma$ is a pair $\gamma=(b, p h)$, where $b$ is a maximal s-connected component of the boundary of a configuration and $p h$ is the function defined on $\partial b$.

It is easy to see that $p h$ takes a constant value on the exterior part of $\partial b$. We shall write $\gamma^{(f)}\left(\gamma^{(3,3)}\right)$ if the value of $p h$ on the exterior part is f.g.s. ((3,3)-g.s.). The set $b$ is called the body of $\gamma$. If $\gamma=(b, p h)$ and $\varphi(b)$ are given one can complete $\varphi(b)$ till the configuration $\varphi_{\gamma}$ on the whole lattice using the boundary conditions in such a way that $B\left(\varphi_{\gamma}\right)=b$.

For any contour $\gamma=(b, p h)$ we put $s \gamma$ to be the contour $(s b, p h)$, where $s b$ is the union of smooth components corresponding to contours of $b$. These smooth components appear after spin-flips destroying all small components. We denote

$$
\begin{gathered}
\partial_{f}(b)=\{z \mid z \in \partial b, p h(z)=\text { f.g.s. }\}, \\
\partial_{(3,3)}(b)=\{z \mid z \in \partial b, p h(z)=(3,3) \text {-g.s. }\}, \\
b_{(\text {int })}=\left\{x \mid x \in b, d\left(x,\left(\partial_{f}(b)\right)^{(\text {hor })}>9, d\left(x,\left(\partial_{(3,3)} b\right)^{(\text {hor })}\right)>6\right\} .\right.
\end{gathered}
$$

A contour $\gamma=(b, p h)$ is called smooth if $b=s b$. By definition the least contour $\gamma^{0}=\left(b^{0}, p h\right)$ is smooth. For any smooth contour $\gamma=(b, p h)$ we put $\operatorname{Sm}(\gamma)$ to be equal to the set of all contours $\gamma_{1}=\left(b_{1}, p h\right)$ which after the spin-flips give the smooth contour $\gamma$. Also

$$
\begin{aligned}
V(\gamma)= & V(b)=\left\{x=\left(x_{1}, x_{2}, x_{3}\right) \in \mathbb{Z}^{3} \mid \partial Q^{\text {(hor) }}(y)\right. \\
\cap & \partial b^{(\text {hor })} \neq \emptyset \text { for some } y=\left(x_{1}, x_{2}, z\right) \in b, \\
& \left.p h\left(\partial Q^{\text {(hor })}(y) \cap \partial b^{\text {(hor) }}\right)=\text { f.g.s., }\left|x_{3}-z\right| \leqq 10\right\} \\
\cup & \left\{x=\left(x_{1}, x_{2}, x_{3}\right) \in \mathbb{Z}^{3} \mid \partial Q^{\text {(hor) }}(y) \cap \partial b^{\text {(hor) }} \neq \emptyset\right. \\
& \text { for some } y=\left(x_{1}, x_{2}, z\right) \in b, p h\left(\partial Q^{\text {(hor) }}(y) \cap \partial b^{\text {(hor) }}\right) \\
= & \left.(3,3)-\text { g.s., }\left|x_{3}-z\right| \leqq 12\right\} \cup b .
\end{aligned}
$$

We shall define now contour models appropriate for the ANNNI model (see the usual case in [6]). Let be given a finite set $U \subset \mathbb{Z}^{3}$. We introduce ensembles $\vartheta^{(f)}(U)\left(\vartheta^{(3,3)}(U)\right)$ whose elements are formal configurations $\left\{\gamma_{i}\right\}$ of mutually disjoint contours $\gamma_{i}^{(f)}\left(\gamma_{i}^{(3,3)}\right)$ belonging to $U$. The distance between any pair of contours is more than 38 .

Remark. Each contour can occupy only a position compatible with its boundary conditions $p h$. This is important for the (3,3)-phase. 
Suppose that functions $F^{(f)}, F^{(3,3)}$ are defined on contours $\gamma^{(f)}, \gamma^{(3,3)}$ respectively taking the same values on congruent contours. We put the statistical weight of a configuration of contours $\left\{\gamma_{i}\right\}$ to be equal to

$$
\begin{gathered}
W\left(\left\{\gamma_{i}^{(f)}\right\}\right)=\exp \left\{-\sum_{i} F^{(f)}\left(\gamma_{i}^{(f)}\right)\right\}, \\
W\left(\left\{\gamma_{i}^{(3,3)}\right\}\right)=\exp \left\{-\sum_{i} F^{(3,3)}\left(\gamma_{i}^{(3,3)}\right)\right\} .
\end{gathered}
$$

The corresponding partition functions are

$$
\Xi\left(U \mid F^{(f)}\right)=\sum_{\left\{\gamma_{i}^{(f)\}}\right.} W\left(\left\{\gamma_{i}^{(f)}\right\}\right), \quad \Xi\left(U \mid F^{(3,3)}\right)=\sum_{\left\{\gamma_{i}^{(3,3)}\right\}} W\left(\left\{\gamma_{i}^{(3,3)}\right\}\right) .
$$

In the usual cases one assumes that $F^{(f)}\left(\gamma^{(f)}\right) \geqq$ const $|b|, F^{(3,3)}\left(\gamma^{(3,3)}\right) \geqq$ const $|b|$ and const is sufficiently large (see [6]). This gives a possibility to present the logarithms of partition functions as sums of two terms where the first one is proportional to $|U|$ while the second one proportional to $|\partial U|$ is a remainder term with nice propoerties (see [6]). We shall see that this main property of contour models remains valid under much more mild assumptions concerning $F^{(f)}, F^{(3,3)}$.

Now we formulate these assumptions. We omit the indices " $f$ " and " $(3,3)$." It means that the formulations are similar in both cases.

\section{Assumptions Concerning $F$}

Let $\gamma$ be a contour, $\partial(s b)^{(\text {ver) }}$ be a vertical boundary of $s b, s \gamma=(s b, p h)$, and $s \gamma$ is the smooth contour corresponding to $\gamma$. For any vertical face $z$ we denote by $T_{0}(z)$ the set of contours $\gamma=(b, p h)$ such that $\partial b^{\text {(ver) }}$ consists of a single connected component and $z \in \partial b^{\text {(ver) }}$. It means that $\gamma$ is completely defined by the boundary condition and $\partial b^{\text {(ver) }}$. If the boundary condition is fixed we shall write $\partial b^{(\mathrm{ver})} \in T_{0}(z)$.

The functional

$$
F(\gamma)=F_{1}(\gamma)+F_{2}(\gamma),
$$

where

1) $F_{1}(\gamma)>K_{1}\left|\partial(s b)^{(\mathrm{ver})}\right|$ for a constant $K_{1}>0$;

2) $F_{2}(\gamma)>-d_{1}^{(1)}|b|$ for a constant $d_{1}^{(1)}>0$;

3) for any smooth $\gamma_{1}=\left(b_{1}, p h\right)$ and any

$$
\begin{aligned}
& \ln \left[\sum_{\gamma=(b, p h) \mid \gamma \in S m\left(\gamma_{1}\right), b(\mathrm{int}) \subset W} \exp \left\{-F_{2}(\gamma)\right\}\right] \\
& \leqq d_{1}^{(1)}\left|b_{1}(\mathrm{int})\right|-d_{1}^{(2)}\left(\left|b_{1} \backslash b_{1}(\mathrm{int})\right|\right) \\
&+\frac{1}{N} \exp \left\{-F\left(\gamma^{(0)}\right)\right\} \cdot\left|\left(V\left(b_{1}\right) \backslash b_{1}(\mathrm{int})\right) \cap W\right|
\end{aligned}
$$

for the same constant $d_{1}^{(1)}$ and a constant $d_{1}^{(2)}>0$; (here $N$ is a period of g.s. $(N=1$ if g.s. $=f, N=6$ if g.s. $=(3,3)))$;

4) if $\gamma=(b, p h)$ and $b=\bigcup b_{i}$, where $b_{i}$ are maximal connected components of $b$ then

$$
F(\gamma)=\sum_{\gamma_{i}=\left(b_{i}, p h\right)} F\left(\gamma_{i}\right)
$$


Contrary to the usual cases $F(\gamma)$ may be negative. The property 3 ) is very important. In fact it requires some estimations of partition functions with summation over contours corresponding to any fixed smooth contour. In our case we shall get needed inequalities during the proof of Theorem B (Sect. 6). We shall use norms

$$
\begin{gathered}
\left\|F_{1}\right\|=\sup _{\gamma=(b, p h)}\left|F_{1}(\gamma)\right| /\left|\partial(s b)^{(\mathrm{ver})}\right|, \\
\left\|F_{2}\right\|=\sup _{\gamma=(b, p h)}\left|F_{2}(\gamma)\right| / / b \mid, \\
\|F\|=\left\|F_{1}\right\|+\left\|F_{2}\right\| .
\end{gathered}
$$

Theorem A. Assume that

$$
\begin{aligned}
d_{1}^{(1)} \leqq & \frac{1}{N} \exp \left\{-F\left(\gamma^{0}\right)\right\}-\exp \left\{-R_{1} K_{1}\right\} \\
& \cdot \exp \left\{-F\left(\gamma^{0}\right)\right\}-R_{2} \sum_{\substack{\gamma \in T_{0}(z) \\
\gamma \neq \gamma^{0}}} \exp \left\{-K_{1}\left|\partial \gamma^{(\mathrm{ver})}\right|\right\}, \\
d_{1}^{(2)}> & \exp \left\{-R_{1} K_{1}\right\} \exp \left\{-F\left(\gamma^{0}\right)\right\} \\
& -R_{2} \sum_{\substack{\gamma \in T_{0}(z) \\
\gamma \neq \gamma^{0}}} \exp \left\{-K_{1}\left|\partial \gamma^{(\mathrm{ver})}\right|\right\} .
\end{aligned}
$$

There exist absolute constants $K_{0}, R_{1}=R_{1}\left(K_{0}\right), R_{2}=R_{2}\left(K_{0}\right)$ such that for $K_{1}$ $>K_{0}$ one can find a number $a(F)$ for which

$$
\ln \Xi(U \mid F)=a|U|+\Delta(U \mid F) .
$$

The remainder term $\triangle(U \mid F)$ satisfies the inequalities

$\left.\mathrm{a}_{1}\right)|\Delta(U \mid F)| \leqq \varrho \cdot|\partial U| ;$

$\left.a_{2}\right)|\Delta(U \mid \bar{F})-\Delta(U \mid \bar{F})| \leqq \varrho\|\bar{F}-\bar{F}\| \cdot|\partial U|$.

Here

$$
\varrho=\varrho\left(K_{1}, d_{1}^{(1)}\right) \leqq \frac{1}{2} \exp \left\{-R_{1} K_{1}-F\left(\gamma^{0}\right)\right\}
$$

is a constant.

Let a contour $\gamma^{(f)}=(b, p h)$ be given. $O_{s}, s=1,2, \ldots, r$ are interior domains corresponding to $b, \chi\left(O_{s}\right)$ are boundary conditions on $\partial b_{s}$ (int). We introduce another ensemble $\vartheta_{c r}\left(\gamma^{(f)}\right)$ whose points are formal admissible configurations of non-intersecting contours inside $O_{s}$ with the same indices " $f$ " and

$$
\Xi^{(c r)}\left(\gamma^{(f)} \mid F^{(f)}\right)=\exp \left\{-F\left(\gamma^{(f)}\right)\right\} \cdot \prod_{s} \sum_{\left\{\gamma_{i}\right\}} \exp \left\{-\sum_{i} F^{(f)}\left(\gamma_{i}^{(f)}\right)\right\},
$$

where the last sum is taken over all configurations of contours $\left\{\gamma_{i}^{(f)}\right\}$ inside $O_{s}$. Then under the conditions of Theorem A

$$
\ln \Xi^{(c r)}\left(\gamma^{(f)} \mid F^{(f)}\right)=-F^{(f)}\left(\gamma^{(f)}\right)+a \cdot \sum_{s}\left|O_{s}\right|+\Delta^{(c r)}\left(\gamma^{(f)} \mid F^{(f)}\right),
$$

where

$$
\Delta^{(c r)}\left(\gamma^{(f)} \mid F^{(f)}\right)=\sum_{s} \Delta\left(O_{s} \mid F^{(f)}\right)
$$


Therefore $\Delta^{(c r)}$ satisfy the inequalities:

$$
\begin{gathered}
\left|\Delta^{(c r)}\left(\gamma^{(f)} \mid F^{(f)}\right)\right| \leqq \varrho|\partial b|, \\
\left|\Delta^{(c r)}\left(\gamma^{(f)} \mid \bar{F}\right)-\Delta^{(c r)}\left(\gamma^{(f)} \mid \overline{\bar{F}}\right)\right| \leqq \varrho\left[\left\|\bar{F}_{1}-\overline{\bar{F}}_{1}\right\|+\left\|\bar{F}_{2}-\overline{\bar{F}}_{2}\right\|\right] \cdot\left|\partial \gamma^{(f)}\right| .
\end{gathered}
$$

In the same manner one can define $\Xi^{(c r)}\left(\gamma^{(3,3)} \mid F^{(3,3)}\right)$, remainder terms $\Delta^{(c r)}\left(\gamma^{(3,3)} \mid F^{(3,3)}\right)$ with the same properties.

\section{Equations for Contour Functionals for the ANNNI Model}

We shall proceed as in [6]. Let us take a configuration $\varphi$ which coincides at infinity either with a f.g.s. or with a $(3,3)$-g.s. Its boundary consists of some number of maximal s-connected subsets $b_{1}, \ldots, b_{r}$ or of contours $\gamma_{1}=\left(b_{1}, p h\right), \ldots, \gamma_{r}=\left(b_{r}, p h\right)$. For any set $V \subset \mathbb{Z}^{3}$ we put

$$
\begin{aligned}
-H(\varphi(V))= & \sum_{x \in V} \varphi(x)\left[\frac{1}{2} J_{0} \sum_{\left(x, x^{\prime}\right) \in\left(\mathbb{Z}^{3}\right)_{\mathrm{hor}}^{(1)}} \varphi\left(x^{\prime}\right)\right. \\
& \left.+\frac{1}{2} J_{1} \sum_{\left(x, x^{\prime}\right) \in\left(\mathbb{Z}^{3}\right)_{\text {ver }}^{(1)}} \varphi\left(x^{\prime}\right)-\frac{1}{2} J_{2} \sum_{\left(x, x^{\prime}\right) \in\left(\mathbb{Z}^{3}\right)_{\text {ver }}^{(2)}} \varphi\left(x^{\prime}\right)\right] .
\end{aligned}
$$

A contour $\gamma_{i}$ is an outer contour if it is not contained inside any domain bounded by other contours. For any contour $\gamma^{(f)}=\left(b^{(f)}, p h\right)$ we denote by $\mathfrak{A}\left(\gamma^{(f)}\right)$ the set of configurations for which the boundary consists of the single contour $b^{(f)}$ and put

$$
\Xi_{1}\left(\gamma^{(f)} \mid \beta, \delta\right)=\sum_{\varphi \in \mathfrak{Q}\left(\gamma^{(f)}\right)} \exp \left\{-\beta H\left(\varphi\left(b^{f}\right)\right)\right\}
$$

For any smooth contour $\gamma_{1}^{(f)}=\left(b_{1}^{(f)}, p h\right)$ and any set $W \supset b_{1}^{(f)}(\mathrm{int}),\left(W \subset \mathbb{Z}^{3}\right)$ we put

$$
\Xi_{W}^{(s)}\left(\gamma_{1}^{(f)} \mid \beta, \delta\right)=\sum_{\substack{\left.(f)=b_{(b)}^{(f)}, p h h\right) \in S m\left(\gamma_{1}^{(f)}\right)(\text { int }) \subset W \\ \text { (f) }}} \exp \left\{\beta H^{(f)}\left(b^{(f)}\right)\right\} \cdot \Xi_{1}\left(\gamma^{(f)} \mid \beta, \delta\right),
$$

where $H^{(f)}(b)$ is the energy of the ferromagnetic configuration on $b$. In an analogous way we can introduce $\Xi_{1}\left(\gamma^{(3,3)} \mid \beta, \delta\right)$ and $\Xi_{W}^{(s)}\left(\gamma_{1}^{(3,3)} \mid \beta, \delta\right)$. Let $h^{(f)}, h^{(3,3)}$ be the energies per particles for f.g.s. and $(3,3)$-g.s. respectively which are functions of $\delta$.

Theorem B. Let $\delta=J_{1}-2 J_{2}>0, \delta=\delta_{0}(\beta)(1+o(1))$ as $\beta \rightarrow \infty$. There exist absolute constants $d_{2}^{(1)}, 0<d_{2}^{(1)}<1$, and $\mathrm{d}_{2}^{(2)}>0$ not depending on $\beta$ and such that

$$
\begin{gathered}
\ln \Xi_{1}\left(\gamma^{(f)} \mid \beta, \delta\right)=-\beta h^{(f)} \cdot|b|-G_{1}^{(f)}\left(\gamma^{(f)}\right)-G_{2}^{(f)}\left(\gamma^{(f)}\right), \\
\ln \Xi_{1}\left(\gamma^{(3,3)} \mid \beta, \delta\right)=-\beta h^{(f)} \cdot|b|-G_{1}^{(3,3)}\left(\gamma^{(3,3)}\right)-G_{2}^{(3,3)}\left(\gamma^{(3,3)}\right), \\
\ln \Xi_{W}^{(s)}\left(\gamma^{(f)} \mid \beta, \delta\right)=-G_{1, W}^{(f)}\left(\gamma^{(f)}\right)-G_{2, W}^{(f)}\left(\gamma^{(f)}\right), \\
\ln \Xi_{W}^{(s)}\left(\gamma^{(3,3)} \mid \beta, \delta\right)=-G_{1, W}^{(3,3)}\left(\gamma^{(3,3)}\right)-G_{1, W}^{(3,3)}\left(\gamma^{(3,3)}\right), \\
\beta C_{1}\left|\partial(s b)^{(\mathrm{ver})}\right| \leqq G_{1}(\gamma) \leqq \beta C_{2}\left|\partial(s b)^{(\mathrm{ver})}\right|, \\
\beta C_{1}\left|\partial b^{(\mathrm{ver})}\right| \leqq G_{1, W}(\gamma) \leqq \beta C_{2}\left|\partial b^{(\mathrm{ver})}\right|, \\
G_{2}(\gamma) \geqq-d_{2}^{(1)} \exp \left\{-\beta \varepsilon_{1}\right\} \cdot|b|, \\
G_{2, W}(\gamma) \geqq-d_{2}^{(1)} \cdot e^{-\beta \varepsilon_{1}} \cdot\left|b(\mathrm{int})+d_{2}^{(2)} e^{-\beta \varepsilon_{1}}\right| b \backslash b(\mathrm{int}) \mid \\
-e^{-\beta \varepsilon_{1}}|(V(b) \backslash b(\mathrm{int})) \cap W|
\end{gathered}
$$


for some absolute positive constants, $C_{1}, C_{2}$. The absence of the index " $f$ " or " $(3,3)$ " means that the inequalities are valid for both types of contours.

Proof of Theorem B is given in Sect. 6. The presence of $d_{2}^{(1)}$ is very important. If the volume $b$ were occupied by a phase then the corresponding partition function would satisfy a similar inequality with $d_{2}^{(1)}=1, d_{2}^{(2)}=0$. We shall take $d_{2}^{(1)}, d_{2}^{(2)}$ from Theorem $\mathrm{B}$ and use Theorem $\mathrm{A}$ with the constants

$$
d_{1}^{(1)}=d_{2}^{(1)} \exp \left\{-\beta \varepsilon_{1}\right\}, \quad d_{1}^{(2)}=d_{2}^{(2)} \exp \left\{-\beta \varepsilon_{1}\right\} .
$$

We recall the notation $O_{s}$ for domains bounded by $\partial b_{s}$ (int) of any smooth contour $\gamma=(b, p h), O=\bigcup_{s} O_{s}, W(b)=\bigcup_{s} O_{s} \cup b$. Now we introduce the most important partition functions

$$
\begin{aligned}
& \Xi(\gamma \mid \beta, \delta) \\
& =\sum \exp \left\{\beta \sum _ { x \in W ( b ) } \varphi ( x ) \left[\frac{1}{2} J_{0} \sum_{\left(x, x^{\prime}\right) \in\left(\mathbb{Z}^{3}\right)_{\mathrm{hor}}} \varphi\left(x^{\prime}\right)\right.\right. \\
& \left.\left.\quad+\frac{1}{2} J_{1} \sum_{\left(x, x^{\prime}\right) \in\left(\mathbb{Z}^{3}\right)_{\text {ver }}^{(1)}} \varphi\left(x^{\prime}\right)-\frac{1}{2} J_{2} \sum_{\left(x, x^{\prime}\right) \in\left(\mathbb{Z}^{3}\right)_{\text {ver }}^{(2)}} \varphi\left(x^{\prime}\right)\right]\right\} .
\end{aligned}
$$

The exterior sum is taken over such configurations $\varphi(W(b))=(\varphi(b), \varphi(O))$ that $\varphi(b) \in \mathfrak{A}(\gamma)$ and $\varphi(O)$ is compatible with boundary conditions.

Main Hypothesis. The needed curve $\delta=\delta(\beta)$ is uniquely defined by the following assumptions: there exist contour functionals $F^{(f)}, F^{(3,3)}$ satisfying the assumptions of Theorem $A$ and such that

1) $a^{(f)}-\beta h^{(f)}=a^{(3,3)}-\beta h^{(3,3)}$,

2) $\Xi\left(\gamma^{(f)} \mid \beta, \delta\right)=\Xi^{(c r)}\left(\gamma^{(f)} \mid F^{(f)}\right) \exp \left\{h^{(f)} \cdot|W(b)|\right\}$.

$$
\Xi\left(\gamma^{(3,3)} \mid \beta, \delta\right)=\Xi^{(c r)}\left(\gamma^{(3,3)} \mid F^{(3,3)}\right) \exp \left\{h^{(3,3)}|W(b)|\right\} .
$$

The relations 1), 2) are quite similar to analogous relations in [6]. Using them we derive first the equations for contour functionals and then solve 1) for finding $\delta$.

Let us take a contour $\gamma^{(f)}$ with its inner components $O_{s} \subset O\left(\gamma^{(f)}\right)$ and fix outer smooth contours $\gamma_{s l}^{\left(\kappa_{s}\right)}$ inside each $O_{s}$. The index " $\kappa_{s}$ " takes the values " $f$ " or " $(3,3)$ " depending on the boundary condition on $\partial O_{s}=\partial b_{s}$ (int). We can write

$$
\begin{aligned}
& \Xi\left(\gamma^{(f)} \mid \beta, \delta\right) \\
& \quad=\Xi_{1}\left(\gamma^{(f)} \mid \beta, \delta\right) \prod_{s} \sum_{\substack{\left\{\gamma^{\left(\kappa_{s}\right)} \\
s l\right.}} \exp \left\{-\beta g^{\left(\kappa_{s}\right)}\left(\left|O_{s}\right|-\sum_{l}\left|W\left(\gamma_{s l}^{\left(\kappa_{s}\right)}\right)\right|\right)\right\} \cdot \prod_{l} \Xi\left(\gamma_{s l}^{\left(\kappa_{s}\right)} \mid \beta, \delta\right) .
\end{aligned}
$$

Using the main hypothesis we rewrite (3) as follows:

$$
\begin{aligned}
\Xi\left(\gamma^{(f)} \mid \beta, \delta\right) \\
=\Xi_{1}\left(\gamma^{(f)} \mid \beta, \delta\right) \cdot \prod_{s} \exp \left\{-\beta h^{\left(\kappa_{s}\right)}\left|O_{s}\right|\right\} \\
\quad \cdot \sum_{\left\{\gamma_{s l}^{\left.\left(\kappa_{s}\right)\right\}}\right.} \prod_{l} \Xi^{(c r)}\left(\gamma_{s l}^{\left(\kappa_{s}\right)} \mid \beta, \delta\right)=\Xi_{1}\left(\gamma^{(f)} \mid \beta, \delta\right) \cdot \prod_{s} \exp \left\{-\beta h^{\left(\kappa_{s}\right)}\left|O_{s}\right|\right\} \cdot \Xi\left(O_{s} \mid F^{\left(\kappa_{s}\right)}\right) \\
=\Xi_{1}\left(\gamma^{(f)} \mid \beta, \delta\right) \cdot \prod_{s} \exp \left\{\left(-\beta h^{\left(\kappa_{s}\right)}+a^{\left(\kappa_{s}\right)}\right)\left|O_{s}\right|+\Delta\left(O_{s} \mid F^{\left(\kappa_{s}\right)}\right)\right\},
\end{aligned}
$$


or with the help of $-\beta h^{(f)}+a^{(f)}=-\beta h^{(3,3)}+a^{(3,3)}$

$$
\begin{aligned}
\exp \{( & \left.-\beta h^{(f)}+a^{(f)}\right) \cdot|W(b)| \\
& \left.-F^{(f)}\left(\gamma^{(f)}\right)-a^{(f)}|b|+\Delta^{(c r)}\left(\gamma^{(f)} \mid F^{(f)}\right)\right\} \\
= & \Xi_{1}\left(\gamma^{(f)} \mid \beta, \delta\right) \exp \left\{\left(-\beta h^{(f)}+a^{(f)}\right) \cdot \sum_{s}\left|O_{s}\right|+\sum_{s} \Delta\left(O_{s} \mid F^{\left(\kappa_{s}\right)}\right)\right\} .
\end{aligned}
$$

Thus we get

$$
-\ln \Xi_{1}\left(\gamma^{(f)} \mid \beta, \delta\right)-\beta h^{(f)} \cdot\left|b^{(f)}\right|=F^{(f)}\left(\gamma^{(f)}\right)-\Delta^{(c r)}\left(\gamma^{(f)} \mid F^{(f)}\right)+\sum_{s} \Delta\left(O_{s} \mid F^{\left(\kappa_{s}\right)}\right) .
$$

Using Theorem B we get a needed equation in its final form:

$$
\begin{aligned}
& G_{1}^{(f)}\left(\gamma^{(f)}\right)+G_{2}^{(f)}\left(\gamma^{(f)}\right) \\
& \quad=F^{(f)}\left(\gamma^{(f)}\right)-\Delta^{(c r)}\left(\gamma^{(f)} \mid F^{(f)}\right)+\sum_{s} \Delta\left(O_{s} \mid F^{\left(\kappa_{s}\right)}\right)
\end{aligned}
$$

In the same manner we get an analogous equation for $F^{(3,3)}$ :

$$
\begin{aligned}
& G_{1}^{(3,3)}\left(\gamma^{(3,3)}\right)+G_{2}^{(3,3)}\left(\gamma^{(3,3)}\right) \\
& \quad=F^{(3,3)}\left(\gamma^{(3,3)}\right)-\beta\left(h^{(f)}-h^{(3,3)}\right)|b|-\Delta^{(c r)}\left(\gamma^{(3,3)} \mid F^{(3,3)}\right)+\sum_{s} \Delta\left(O_{s} \mid F^{\left(\kappa_{s}\right)}\right) .
\end{aligned}
$$

The existence of solutions of (5), (6) is shown in Sect. 5. In Sect. 7 we discuss the final steps and make some conclusions.

\section{Generalized Contour Models and Proof of Theorem A}

We shall consider only ensembles $\vartheta^{(f)}(U)$; the case of $\vartheta^{(3,3)}(U)$ is treated in a similar way. The index " $f$ " is therefore omitted. A contour $\gamma$ is called an outer contour of a configuration $\left\{\gamma_{i}\right\}$ if it is not contained in any inner domain $O_{s}\left(\gamma_{i}\right)$ of another contour. As usual (see $[5,6]$ ), we introduce correlation functions $\pi_{s}\left(\gamma_{1}, \ldots, \gamma_{s} \mid U, F\right)$ $=\pi_{s}\left(\gamma_{1}, \ldots, \gamma_{s}\right)$ which are equal to probabilities of the presence of $s$ outer contours $\gamma_{1}, \ldots, \gamma_{s}, \gamma_{i}=\left(b_{i}, p h\right)$ in a random configuration of contours. We shall derive now correlation equations for $\pi_{s}$ which differ slightly from the usual correlation equations for outer contours (see $[5,6]$ ).

We use the notation $T(z)$ for the set of all contours $\gamma=(b, p h)$ such that $z \in \partial b^{\text {(ver) }}$. Also we put $r_{1}=19$ if $p h=f$ and $r_{1}=18$ if $p h=(3,3)$.

For any outer contour $\gamma=(b, p h)$ we denote by $W(\gamma)$ the set $\left\{x \mid x=\left(x_{1}, x_{2}, x_{3}\right)\right.$ $\notin b$; there exists a point $y=\left(y_{1}, y_{2}, y_{3}\right) \subset \partial b^{(\text {hor })}$ for which $y_{1}=x_{1}, y_{2}=x_{2},\left|x_{3}-y_{3}\right|$ $\leqq 38\}$. Put

$$
\begin{gathered}
W_{1}\left(\gamma_{1}, \ldots, \gamma_{p}\right)=\bigcup_{i=1}^{p}\left(W\left(\gamma_{i}\right) \cup b_{i}\right), \\
W_{2}\left(\gamma_{1}, \ldots, \gamma_{p}\right)=W_{1}\left(\gamma_{1}, \ldots, \gamma_{p}\right) \cup\left(\bigcup_{x \mid b^{(0)}(x) \cap W_{1}\left(\gamma_{1}, \ldots, \gamma_{p}\right) \neq \emptyset} b^{(0)}(x)\right), \\
W_{3}\left(\gamma_{1}, \ldots, \gamma_{p}\right)=W_{1}\left(\gamma_{1}, \ldots, \gamma_{p}\right) \cup\left\{x \mid b^{(0)}(x) \cap W_{1}\left(\gamma_{1}, \ldots, \gamma_{p}\right) \neq \emptyset\right\}, \\
W_{i}^{(m)}\left(\gamma_{1}, \ldots, \gamma_{p}\right)=\left\{x \mid x \in W_{i}\left(\gamma_{1}, \ldots, \gamma_{p}\right), d\left(\partial W_{i}\left(\gamma_{1}, \ldots, \gamma_{p}\right), x\right) \leqq m\right\}, \\
i=1,2,3 .
\end{gathered}
$$


We have

$$
\begin{aligned}
\pi_{p}\left(\gamma_{1}, \ldots, \gamma_{p} \mid U, F\right) & =\frac{\Sigma_{l}^{(1)} \prod_{l} w\left(\gamma_{l}\right)}{\Xi(U, F)} \\
& =\frac{\Xi_{1}\left(\gamma_{1}, \ldots, \gamma_{p}\right) \cdot \Sigma^{(2)} \prod_{l} w\left(\gamma_{l}\right)}{\Xi(U, F)} \cdot \frac{\exp \left\{-\sum_{i=1}^{p} F\left(\gamma_{i}\right)\right\}}{\Xi_{1}\left(\gamma_{1}, \ldots, \gamma_{p}\right)} .
\end{aligned}
$$

Here the sum $\Sigma^{(1)}$ is taken over all configurations containing outer contours $\gamma_{1}, \ldots, \gamma_{p} ; \sum^{(2)}$ is the sum over all configurations not containing outer contours $\gamma_{1}, \ldots, \gamma_{p}$ and containing only contours which do not intersect and not encircle any of contours $\gamma_{1}, \ldots, \gamma_{p} ; \Xi_{1}\left(\gamma_{1}, \ldots, \gamma_{p}\right)$ is the partition function over all configurations of contours $\gamma=(b, p h)$ such that the following two properties are valid:

$\left.\mathrm{a}_{1}\right) b \subset W_{2}\left(\gamma_{1}, \ldots, \gamma_{p}\right)$,

$\left.\mathrm{a}_{2}\right)$ if $b \cap W_{1}^{(38)}\left(\gamma_{1}, \ldots, \gamma_{p}\right) \neq \emptyset$ then $\gamma=(b, p h)$ is the least contour.

Let us explain why we need the volume $W_{1}^{(38)}$. Assume for simplicity that $p=1$ and we are dealing with a single contour $\gamma_{1}$. Then in view of our definitions in the 38 -neighbourhood of $\partial b_{1}^{\text {(hor) }}$ the configuration is fixed and the points where spinflips produce the least contours $s$-connected with $\gamma_{1}$ must belong to $W_{2}\left(\gamma_{1}\right)$. We put now

$$
\lambda\left(\gamma_{1}, \ldots, \gamma_{p}\right)=\Xi_{1}^{-1}\left(\gamma_{1}, \ldots, \gamma_{p}\right) \exp \left\{-\sum_{i=1}^{p} F\left(\gamma_{i}\right)\right\}
$$

Lemma 1. There exists an absolute constant $R_{0}^{(1)}$ such that

$$
\begin{gathered}
\lambda\left(\gamma_{1}, \ldots, \gamma_{p}\right) \leqq \exp \left\{-\sum_{i=1}^{p} F\left(\gamma_{i}\right)-\frac{1}{N} \exp \left\{-F\left(\gamma^{(0)}\right)\right\}\right. \\
\left.\cdot\left|W_{3}\left(\gamma_{1}, \ldots, \gamma_{p}\right)\right|\left(1+\varrho\left(K_{1} ; \gamma_{1}, \ldots, \gamma_{p}\right)\right)\right\},
\end{gathered}
$$

where

$$
\left|\varrho\left(K_{1} ; \gamma_{1}, \ldots, \gamma_{p}\right)\right| \leqq \exp \left\{-R_{1}^{(1)} K_{1}\right\} .
$$

Proof. We restrict ourselves by summation in $\Xi_{1}$ over configurations consisting only of the least contours. The result of this summation can be written in the form

$$
\exp \left\{\frac{1}{\mathrm{~N}} \exp \left\{-F\left(\gamma^{(0)}\right)\right\} \cdot\left|W_{3}\left(\gamma_{1}, \ldots, \gamma_{p}\right)\right|\left(1+\varrho\left(K_{1} ; \gamma_{1}, \ldots, \gamma_{p}\right)\right)\right\}
$$

where $\varrho\left(K_{1} ; \gamma_{1}, \ldots, \gamma_{p}\right)$ satisfies the needed estimation. Q.E.D.

Lemma 2. Let $\gamma=(b, p h)$ be a smooth contour such that $b$ is connected. Put

$$
\lambda_{1}(\gamma)=\lambda_{1}(b)=\exp \left\{-K\left|\partial b^{(\mathrm{ver})}\right|-d_{2}|b|\right\},
$$

where $K, d_{2}$ are constants.

There exist constant $K_{0}, R=R\left(K_{0}\right)$ such that if

$$
K>K_{0}, \quad d_{2}>R \sum_{\gamma \in T_{0}(z), \gamma \neq \gamma^{(0)}} \exp \left\{-K\left|\partial b^{(\mathrm{ver})}\right|\right\}
$$


then

$$
\sum_{\substack{\gamma \in T(z) \\ \gamma \neq \gamma(0)}} \lambda_{1}(\gamma) \leqq \exp \left\{-R_{1} K\right\} \cdot \exp \left\{-F\left(\gamma^{(0)}\right)\right\}
$$

where $R_{1}$ is an absolute constant.

Proof. Firstly we shall consider a simpler model where one can understand better the essence of the situation and of our arguments and then shall make necessary additional remarks. Twice during the proof we shall use a trick which we have learned from Zahradnik [7].

In the simplified model we consider Ising type contours which are connected volumes. The statistical weight of any such contour $b$ is equal to

$$
\lambda_{1}(b)=\exp \left\{-K\left|\partial b^{(\mathrm{ver})}\right|-D|b|\right\},
$$

where

$$
D=R \sum_{b \in T_{0}(z), b \neq b_{0}(z)} \exp \left\{-K\left|\partial b^{(\mathrm{ver})}\right|\right\},
$$

$R$ is a constant. Here $b_{0}(z)$ is the least contour for which $\left|b_{0}(z)\right|=1$. A contour $b$ is smooth if either it is the least contour or $\left|\partial b_{i}^{(\mathrm{ver})}\right|>4$ for all vertical components of the boundary.

We fix $\overline{\partial b}$ and $B$ and consider first the sum $\sum \lambda_{1}(b)$ over such $b$ for which $\partial b($ ext $)$ $=\overline{\partial b}$ and $|b|=B$. We denote by $\bar{b}$ the connected bounded set, for which $\partial(\bar{b})=\overline{\partial b}$, $B_{1}=|\bar{b}|$. We introduce an auxiliary model. For every finite set $O$ we denote by $\mathfrak{L}(O)$ an ensemble whose points are formal configurations of disjoint sets $a_{i}$, where each $a_{i}$ is a boundary of a simply connected set, $a_{i}=\partial \mathscr{A}_{i}, a_{i} \cap O \neq \emptyset$, and put

$$
\lambda_{1}\left(\left\{a_{i}\right\}\right)=\prod_{i} \exp \left\{-K\left|a_{i}^{(\mathrm{ver})}\right|\right\}, \quad \Xi_{\mathfrak{l}}(O)=\sum_{\left\{a_{i}\right\}} \lambda_{1}\left(\left\{a_{i}\right\}\right) .
$$

Here $a_{i}^{(\mathrm{ver})}$ is the set of vertical faces of $a_{i}$ which determines uniquely $a_{i}$. This model can be investigated by the usual methods of the theory of contour models. In particular, one can use Kirkwood-Salzburg equations for correlation functions of $a_{i}$ and investigate the behaviour of $\Xi_{\mathfrak{Q}}(O)$ for large $O$ provided that $K$ is large enough. For such $K$ one can find $\alpha(K)$ for which

$$
\Xi_{\mathfrak{L}}(O)=\exp \left\{\alpha(K) \cdot|O|+\alpha_{1}(K, O)|\partial O|\right\},
$$

where

$$
\left|\alpha_{1}(K, O)\right| \leqq \exp \{-K r+\text { const }\},
$$

and $r$ is the cardinality of the least possible $\left|a_{i}^{(\mathrm{ver})}\right|$. Now we have

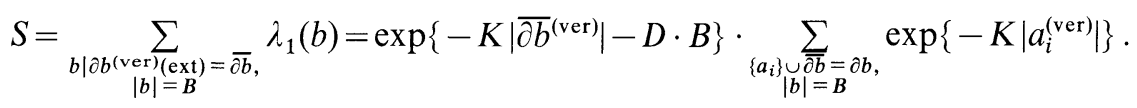

The last sum is estimated in the following way:

$$
\begin{aligned}
& \sum_{\left\{a_{i}\right\} \cup \overline{\overline{\partial b}}=\partial b} \exp \left\{-K\left|a_{i}^{(\mathrm{ver})}\right|\right\}=\exp \left\{-\alpha(K)\left(B_{1}-B\right)\right\} \\
& \cdot \sum_{\left\{a_{2}\right\}} \prod_{i} \exp \left\{-K\left|a_{i}^{(\mathrm{ver})}\right|\right\} \cdot \Xi_{\mathfrak{L}}\left(\mathscr{A}_{i}\right) \cdot \exp \left\{-\alpha_{1}\left(K, \mathscr{A}_{i}\right) \cdot\left|a_{i}\right|\right\} \\
& \leqq \\
& \quad \exp \left\{-\alpha(K)\left(B_{1}-B\right)+\max _{i}\left|\alpha_{1}\left(K, \mathscr{A}_{i}\right)\right| B\right\} \cdot \Xi_{\mathfrak{Q}}(\bar{b}) \\
& =\exp \left\{\alpha(K) \cdot B+2 \max _{\mathscr{A}}\left|\alpha_{1}(K, \mathscr{A})\right| \cdot B\right\}
\end{aligned}
$$


Thus

$$
S \leqq \exp \left\{-K\left|\overline{\partial b}^{(\text {ver })}\right|-D_{1} B\right\}
$$

where

$$
D_{1}=D-2 \alpha(K)-2 \max _{\mathscr{A}}\left|\alpha_{1}(K, \mathscr{A})\right|
$$

Now we have to consider in $a$ more detail the structure of $\overline{\partial b^{(v e r)}}(\mathrm{ext})$. Each $\overline{\partial b}^{\text {(ver) }}$ (ext) consists of several connected vertical components $\overline{\partial b}_{i}^{\text {(ver) }}$ (ext). It is an appropriate moment to introduce several notions. A vertical component $a$ is a connected set of vertical faces such that each vertical edge belongs to an even number of faces. The space of all vertical components is denoted by $A$, the space of $a$ belonging to a set $O$ is denoted by $A(O)$. Each $\overline{\partial b}^{(\mathrm{ver})}$ is a union of a finite number of $a \in A, \overline{\partial b}^{\text {(ver) }}=\bigcup_{i} a_{i}$.

Having $a$ we can complete it in a unique way to the boundary of a connected set $[a]$ adding some number of connected horizontal components $\eta_{j}=\eta_{j}(a)$ in such a way that $\partial \eta_{j}$ is contained in the set of horizontal edges of faces of $a$. Thus $a \cup \bigcup_{j} \eta_{j}$ $=\partial[a], \partial([a])^{(\mathrm{ver})}=a$. The set $[a]$ will be called an interior of the vertical component a.

Assume that $z$ is a vertical face, $z \in a_{0} \subset \overline{\partial b}^{\text {(ver) }}$. We shall call $a_{0}$ a vertical component of the zeroth level. By induction, suppose that vertical components of $\overline{\partial b}^{\text {(ver) }}$ of the $\ell^{\text {th }}$ level are defined. Denote them by $a_{\ell, i}, 1 \leqq i \leqq I_{\ell}$. We construct $\eta_{j}\left(a_{\ell, i}\right)$ and define the components of the $(\ell+1)^{\text {th }}$ level as those components of $\frac{\partial}{\partial b^{(v e r)}}$ which intersect $\eta_{j}\left(a_{\ell, i}\right)$ and are not components of the previous levels. The level of a component $a_{i}$ is denoted by $\ell\left(a_{i}\right), \ell\left({\overline{\partial b^{(v e r)}}}^{\text {(v) }}=\max _{a_{1} \subset \bar{\partial} b^{\text {(ver) }}} \ell\left(\bar{a}_{i}\right)\right.$. If we are given vertical components $a_{\ell, i}, 1 \leqq i \leqq I_{\ell}, \ell=\ell_{0}$, we can construct $\overline{\partial b}_{\ell_{0}}=\bigcup_{\ell=0}^{\ell_{0}} \bigcup_{i=1}^{I_{2}} a_{\ell, i}$ and consider $b, \partial b=\overline{\partial b}_{\ell_{0}}$. We have obviously $\ell\left(\overline{\partial b}_{\ell_{0}}^{(\text {ver })}\right)=\ell_{0}$. We denote $\partial T_{\ell_{0}}(z)$ the set of $\overline{\partial b}, z \in \overline{\partial b}$ and $\ell(\overline{\partial b})=\ell_{0}$. Now we fix $a_{0}$ and have to estimate the sum

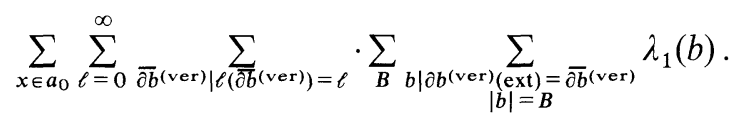

The last two sums were in fact already estimated. Indeed, if we denote by $\mathscr{H}\left(\overline{\partial b}^{\text {(ver) }}\right)$ the number of horizontal faces of $\overline{\partial b}$ which correspond uniquely to $\overline{\partial b}^{\text {(ver) }}$, then

$$
\frac{1}{2} \mathscr{H}\left(\partial b^{(\text {ver })}\right) \leqq B \leqq\left|\partial b^{(\text {ver })}\right|^{2},
$$

and using (12) we get

$$
\begin{aligned}
& \sum_{B} \sum_{b \mid \partial b^{(\text {ver })}(\text { ext })=\bar{\partial} \bar{b}^{(\text {ver })},|b|=B} \lambda_{1}(b) \\
& \leqq \sum_{B} \exp \left\{-K\left|\overline{\partial b}^{(\text {ver })}\right|-D_{1} B\right\} \\
& \leqq\left|\overline{\partial b}^{\text {(ver })}\right|^{2} \cdot \exp \left\{-K\left|\overline{\partial b}^{\text {(ver })}\right|-\frac{1}{2} D_{1} \mathscr{H}\left({\overline{\partial b^{(v e r)}}}^{(\text {v }}\right)\right\} \\
& \leqq \exp \left\{-K^{(1)}\left|\overline{\partial b^{(v e r)}}\right|-\frac{1}{2} D_{1} \mathscr{H}\left(\overline{\partial b}^{(\text {ver })}\right)\right\} \text {, }
\end{aligned}
$$


where

$$
K^{(1)}=K_{1}-2 \max _{t \geqq 1} t^{-1} \ln t .
$$

Now we have a simpler sum,

$$
S=\sum_{x \in a_{0}} \sum_{\ell=0}^{\infty} \sum_{\overline{\partial b^{(v e r) ~}} \mid \ell\left(\partial b^{\text {(ver })}\right)=\ell} \cdot \exp \left\{-K^{(1)}\left|\overline{\partial b}^{(\text {ver })}\right|-\frac{1}{2} D_{1} \mathscr{H}\left(\overline{\partial b}^{(\text {ver })}\right)\right\} .
$$

We shall estimate it by induction from large to small $\ell$. Starting with an arbitrarily large $\mathscr{L}$ we assume that the summation over all contours $b$, for which $\ell_{0}<\ell(b)$ $\leqq \mathscr{L}$ is already performed, and the result is

$$
S_{\ell_{0}}=\sum_{\overline{\partial b} \in \partial T_{\ell_{0}}(z)} \cdot \exp \left\{-K^{(1)} \sum_{\ell=0}^{\ell_{0}-1} \sum_{i=1}^{I_{\ell}}\left|a_{\ell, i}\right|-K^{(2)} \sum_{i=1}^{I_{\ell_{0}}}\left|a_{\ell_{0}, i}\right|-\frac{1}{2} D_{1} \mathscr{H}(\overline{\partial b})\right\} .
$$

Here

$$
K^{(2)}=K^{(1)}-2 \max _{t \geqq 1} t^{-1} \ln t .
$$

We shall use again Zahradnik's trick. Let $\eta_{k}$ be a horizontal component of $a_{\ell_{0}-1, j}$. We consider an ensemble $\mathfrak{B}_{1}\left(\eta_{k}\right)$ whose elements are mutually disjoint vertical components $a_{\ell_{0}, j}$ such that $\left[a_{\ell_{0}, j}\right] \cap \eta_{k} \neq \emptyset$. We shall estimate the sum

$$
W=\sum_{\left\{a_{\ell_{0}}, j\right\} \in \mathfrak{S}_{1}\left(\eta_{k}\right)} \exp \left\{-K^{(2)} \sum_{j}\left|a_{\ell_{0}, j}\right|-\frac{1}{2} D_{1} M\right\},
$$

where $M$ is the cardinality of the set of horizontal faces of $\eta_{k}$ belonging to $\overline{\partial b}^{\text {(hor) }}$, and thus lying outside all $\left[a_{\ell_{0}, j}\right]$. We can write

$$
W=\sum_{M} \exp \left\{-\frac{1}{2} D_{1} M\right\} \cdot \sum \exp \left\{-K^{(2)} \sum_{j}\left|a_{\ell_{0}, j}\right|\right\}
$$

where the inner summation goes over all configurations $\left\{a_{\ell_{0}, j}\right\}$ with the fixed value of $M$.

For any set $O \subset \eta_{k}$ and $K>0$ we put

$$
\Xi_{\mathfrak{Q}_{1}}(O)=\sum_{\substack{\left\{a_{\ell_{0}}, j\right\} \subset \mathfrak{Q}_{1}\left(\eta_{k}\right) \\\left[a_{\ell_{0}}, j\right] \cap \eta_{k} \subset O}} \exp \left\{-K \sum_{j}\left|a_{\ell_{0}, j}\right|\right\} .
$$

For sufficiently large $K$ one can find $\bar{\alpha}(K)$ and $\bar{\alpha}_{1}(K, O)$ such that

$$
\ln \Xi_{\mathfrak{\Omega}_{1}}(O)=\bar{\alpha}(K)|O|+\bar{\alpha}_{1}(K, O) \cdot|\partial O|,
$$

where $\bar{\alpha}(K), \bar{\alpha}_{1}(K, O) \leqq$ const $e^{-K r}$ as $K \rightarrow \infty$, and $r$ is the least possible value of $\left|a_{\ell_{0}, j}\right|$. In all these arguments we assumed that all $a_{\ell_{0}, j} \subset \mathfrak{I}_{1}\left(\eta_{k}\right)$. But there can be also components $a_{\ell_{0}, j}$ which encircle connected components of $\eta_{k}$. The summation over such configurations only changes slightly the remainder term in (15). We shall assume later that this correction is already present in (15). Then in (14)

$$
\begin{aligned}
\sum_{\left\{a_{\ell_{0}}, j\right\}} & \exp \left\{-K^{(2)} \sum_{j}\left|a_{\ell_{0}, j}\right|\right\} \leqq \exp \left\{-\bar{\alpha}\left(K^{(2)}\right)\left[\left|\eta_{k}\right|-M\right]\right\} \\
& \cdot \sum_{\left\{a_{\ell_{0}}, j\right\}} \prod_{j} \exp \left\{-K^{(2)}\left|a_{\ell_{0}, j}\right|\right\} \cdot \Xi_{\mathfrak{S}_{1}}\left(\left[a_{\ell_{0}, j}\right] \cap \eta_{k}\right) \\
& \cdot \exp \left\{-\bar{\alpha}_{1}\left(K^{(2)},\left[a_{\ell_{0}, j}\right] \cap \eta_{k}\right) \cdot\left|\partial\left(\left[a_{\ell_{0}, j}\right] \cap \eta_{k}\right)\right|\right\} \\
\leqq & \exp \left\{-\bar{\alpha}\left(K^{(2)}\right) \cdot\left[\left|\eta_{k}\right|-M\right]+\max \left|\bar{\alpha}_{1}\left(K^{(2)},\left[a_{\ell_{0}, j}\right] \cap \eta_{k}\right)\right| \cdot M\right\} \\
& \cdot \Xi_{\mathfrak{I}_{1}}\left(\eta_{k}\right)=\exp \left\{\bar{\alpha}_{2}\left(K^{(2)}\right) \cdot M\right\},
\end{aligned}
$$


where

$$
\bar{\alpha}_{2}\left(K^{(2)}\right)=\bar{\alpha}\left(K^{(2)}\right)+2 \max \left|\bar{\alpha}_{1}\left(K^{(2)},\left[a_{\ell_{0}, j}\right] \cap \eta_{k}\right)\right| .
$$

Assuming that $\frac{1}{2} D_{1} \geqq \bar{\alpha}_{2}\left(K^{(2)}\right)$, we have

$$
W \leqq \sum_{M} \exp \left\{\left(-\frac{1}{2} D_{1}+\bar{\alpha}_{2}\left(K^{(2)}\right) M\right\} \leqq \sum_{i}\left|a_{\ell_{0}-1, i}\right|^{2}\right.
$$

Finally

$$
\begin{aligned}
S_{\ell_{0}-1} \leqq & \sum_{\partial \bar{b} \in \partial T_{\ell_{0}-1}(z)} \\
& \cdot \exp \left\{-K^{(1)} \sum_{\ell=0}^{\ell_{0}-2} \sum_{i=1}^{I_{\ell}}\left|a_{\ell, i}\right|-K^{(2)} \sum_{i=1}^{I_{\ell_{0}-1}}\left|a_{\ell_{0}-1, i}\right|-\frac{1}{2} D_{1} \mathscr{H}(\overline{\partial \bar{b}})\right\} .
\end{aligned}
$$

Thus we passed from $\ell_{0}$ to $\ell_{0}-1$. Therefore for $\ell_{0}=0$, the sum $S_{0}$ $\leqq \sum_{a_{0}} \exp \left\{-K^{(2)}\left|a_{0}\right|\right\}$. The number of all possible components $a_{0}$ with $\left|a_{0}\right|=m$ is not more than $C^{m}$, where $C$ is an absolute constant. This gives the final result provided that

$$
D \geqq 2 \alpha(K)+2 \max _{0}\left|\alpha_{1}(K, O)\right|+2\left[\bar{\alpha}\left(K^{(2)}\right)+\max _{o}\left|\bar{\alpha}_{1}\left(K^{(2)}, O\right)\right|\right]
$$

One easily finds that for sufficiently large $K$ the numbers $D \sim R \exp \{-K r\}$, $\alpha(K), \max _{o}\left|\alpha_{1}(K, O)\right|, \bar{\alpha}\left(K^{(2)}\right), \max _{o}\left|\bar{\alpha}_{1}\left(K^{(2)}, O\right)\right| \leqq$ const $\exp \{-K r\}$, where $r$ is the least possible value of $\left|a_{0}\right| \in T_{0}(z)$ and const is an absolute constant. Thus if $R$ is sufficiently large the inequality (16) is valid.

Our arguments can be extended without any difficulties to more complicated situations such as contour models introduced in Sect. 3. The final conclusion and the result will be the same provided that $K$ and $R$ are sufficiently large. Q.E.D.

We shall say that a contour $\gamma=(b, p h)$ has the property A if the following is true:

For the $\operatorname{strip} S=W_{1}^{(38)}\left(\gamma_{1}, \ldots, \gamma_{p}\right)$ the intersection $b \cap S \neq \emptyset$, and there exists a configuration of the least contours

$$
\left\{\gamma^{(0)}\left(x_{i}\right)=\left(b^{(0)}\left(x_{i}\right), p h\right)\right\}, \quad i=1, \ldots, q(\gamma),
$$

such that

$$
b=\bigcup_{i=1}^{q(\gamma)} b^{(0)}\left(x_{i}\right) \cup(b \backslash S), \quad x_{i} \in W_{3}^{\left(38+2 r_{1}\right)}\left(\gamma_{1}, \ldots, \gamma_{p}\right) .
$$

We denote by $\gamma_{x}$ any contour containing $x \in \mathbb{Z}^{3}$.

Let us consider the fraction

$$
Q=\frac{\Xi_{1}\left(\gamma_{1}, \ldots, \gamma_{p}\right) \cdot \sum^{(2)} \prod_{i} w\left(\gamma_{i}\right)}{\Xi(U \mid F)} .
$$

It is easy to see that

$$
Q=E\left\{\left[\sum_{M \subset S} \prod_{x \in M} \sum_{\gamma_{x}^{A}} \chi_{\gamma_{x}^{A}} \cdot f\left(\gamma_{x}^{A}\right) \prod_{x \in S \backslash M} \prod_{\gamma_{x}^{\prime}}\left(1-\chi_{\gamma_{x}^{\prime}}\right)\right] \prod_{\bar{\gamma}}\left(1-\chi_{\bar{\gamma}}\right)\right\} .
$$


Here $\gamma_{x}^{A}$ is the notation for the contour having the property $A$ and containing $x, \gamma$ is a contour encircling at least one of the contours $\gamma_{1}, \ldots, \gamma_{p}$;

$$
\begin{aligned}
f\left(\gamma^{A}\right)= & \exp \left\{F\left(\gamma^{A}\right)-F\left(\hat{\gamma}^{A}\right)\right. \\
& \left.-\sum_{j \mid b^{(0)}\left(x_{j}\right) \cap \partial W_{1} \neq \emptyset} F\left(\gamma^{(0)}\left(x_{j}\right)\right)\right\}, \quad \gamma^{A}=(b, p h), \\
& \hat{\gamma}^{A}=(\hat{b}, p h), \quad \hat{b}=b \bigcup_{j} b^{(0)}\left(x_{j}\right) \cap S, \chi_{\gamma}
\end{aligned}
$$

is the indicator for the set of configurations having an outer contour $\gamma, \gamma_{x}^{\prime}$ is a contour intersecting $S$ and is not the least contour, $E$ means the expectation with respect to the ensemble $\vartheta(U)$.

The last expression shows that $Q$ can be written as a linear combination of correlation functions which gives us the desired system of correlation equations:

$$
\begin{aligned}
\pi_{p}\left(\gamma_{1}, \ldots, \gamma_{p}\right) \\
=\lambda\left(\gamma_{1}, \ldots, \gamma_{p}\right)\left[1+\sum_{\gamma_{1}^{A}, \ldots, \gamma_{r}^{A}} \prod_{i=1}^{r} f\left(\gamma_{i}^{A}\right)\left(\sum_{\gamma_{1}^{\prime}, \ldots, \gamma_{m}^{\prime}}(-1)^{m}\right.\right. \\
\quad \cdot \pi_{r+m}\left(\gamma_{1}^{A}, \ldots, \gamma_{r}^{A}, \gamma_{1}^{\prime}, \ldots, \gamma_{m}^{\prime}\right) \\
\left.\left.\quad+\sum_{\bar{\gamma}_{1}, \ldots, \bar{\gamma}_{\ell}}(-1)^{\ell} \pi_{r+m+\ell}\left(\gamma_{1}^{A}, \ldots, \gamma_{r}^{A}, \gamma_{1}^{\prime}, \ldots, \gamma_{m}^{\prime}, \bar{\gamma}_{1}, \ldots, \bar{\gamma}_{\ell}\right)\right)\right] .
\end{aligned}
$$

We introduce the following norm in the space of sequences $\pi=\left\{\pi_{p}\left(\gamma_{1}, \ldots, \gamma_{p}\right)\right\}$ :

$$
\|\pi\|=\sup _{p,\left\{\gamma_{1}, \ldots, \gamma_{p}\right\}} \frac{\left|\pi_{p}\left(\gamma_{1}, \ldots, \gamma_{p}\right)\right|}{\bar{\lambda}\left(\gamma_{1}, \ldots, \gamma_{p}\right)}
$$

where

$$
\begin{aligned}
\bar{\lambda}\left(\gamma_{1}, \ldots, \gamma_{p}\right)= & \lambda\left(\gamma_{1}, \ldots, \gamma_{p}\right) \exp \left\{\frac{1}{N} \exp \left\{-F\left(\gamma^{(0)}\right)-c K_{1}\right\}\right. \\
& \cdot\left|\left(\partial W_{3}\left(\gamma_{1}, \ldots, \gamma_{p}\right)\right)^{(\text {hor })}\right| \\
& \left.+\frac{D}{N} \exp \left\{-F\left(\gamma^{(0)}\right)\right\} \cdot \sum_{i=1}^{p}\left|\partial\left(s \gamma_{i}\right)^{(\mathrm{ver})}\right|\right\},
\end{aligned}
$$

$c, D$ are constants.

The system of correlation equations can be written in the form $\pi=\Lambda \pi+\Phi$. Here $\Lambda$ is a linear operator, $\Phi=\left\{\lambda\left(\gamma_{1}, \ldots, \gamma_{p}\right)\right\}$.

Lemma 3. There exist constants $K_{0}, R^{(1)}=R^{(1)}\left(K_{0}\right), R^{(2)}=R^{(2)}\left(K_{0}\right)$ such that for $K_{1}>K_{0}$,

$$
\begin{aligned}
\Sigma^{r, m}= & \Sigma^{r, m}\left(z_{1}, \ldots, z_{r}, z_{1}^{\prime}, \ldots, z_{m}^{\prime}\right)=\sum_{\substack{\gamma_{1}^{A} \in T\left(z_{1}\right), 1 \leqq i \leqq r \\
\gamma_{j}^{\prime} \neq \gamma^{(0)} \in T\left(z_{j}^{\prime}\right), 1 \leqq j \leqq m}} \\
& \cdot \bar{\lambda}\left(\gamma_{1}^{A}, \ldots, \gamma_{r}^{A}, \gamma_{1}^{\prime}, \ldots, \gamma_{m}^{\prime}\right) \cdot f\left(\gamma_{1}^{A}, \ldots, \gamma_{r}^{A}\right) \\
\leqq & \left(\exp \left\{-R^{(1)} K_{1}\right\} \cdot \frac{1}{N} \exp \left\{-F\left(\gamma^{(0)}\right)\right\}+R^{(2)} \sum_{b \in T_{0}(z)} \exp \left\{-K_{1}\left|\partial b^{(\mathrm{ver})}\right|\right\}\right)^{r+m},
\end{aligned}
$$


where

$$
z_{i} \in W_{1}^{(38)}\left(\gamma_{1}, \ldots, \gamma_{p}\right), \quad z_{j}^{\prime} \in W_{1}^{(38)}\left(\gamma_{1}, \ldots, \gamma_{p}\right)
$$

are arbitrary vertical faces.

Proof. We shall write for brevity $f(\Gamma)$ instead of $f\left(\gamma_{1}, \ldots, \gamma_{\ell}\right)$ if $f$ is a function of a configuration $\Gamma=\left\{\gamma_{1}, \ldots, \gamma_{\ell}\right\}$,

$$
\begin{gathered}
W(\Gamma, D, c)=\exp \left\{-c K_{1}\right\}\left|\partial W_{3}^{(\text {hor })}(\Gamma)\right| \\
+D \sum_{\gamma \in \Gamma}\left|\partial(s \gamma)^{(\mathrm{ver})}\right|, \\
\Gamma_{A}^{(r)}=\left\{\gamma_{i}^{A}\right\}_{i=1}^{r}, \quad \Gamma^{(m)}=\left\{\gamma_{j}^{\prime}\right\}_{j=1}^{m}, \\
\Gamma^{(r, m)}=\Gamma_{A}^{(r)} \cup \Gamma^{(m)}, \quad \Gamma_{A, I}^{(r)}=\left\{\gamma_{i}^{A}\right\}_{i \in I},
\end{gathered}
$$

$I$ is a subset of the set $(1, \ldots, r)$,

$$
\begin{aligned}
\Gamma_{A, I}^{(r, m)} & =\Gamma_{A, I}^{(r)} \cup \Gamma^{(m)}, \quad \Gamma_{p}=\left\{\gamma_{1}, \ldots, \gamma_{p}\right\}, \\
\hat{\Gamma}_{A, I}^{(r)} & =\left\{\hat{\gamma}_{i}^{A}\right\}_{i \in I}, \quad \hat{\Gamma}_{A, I}^{(r, m)}=\hat{\Gamma}_{A, I}^{(r)} \cup \Gamma^{(m)} .
\end{aligned}
$$

It is easy to see that if $\hat{\gamma}_{i}^{A}=\gamma^{(0)}$, then

$$
\begin{gathered}
\sum_{\gamma_{i}^{A} \mid \gamma_{i}^{A}=\gamma^{(0)}} \exp \left\{-F\left(\gamma_{i}^{A}\right)\right\} \cdot f\left(\gamma_{i}^{A}\right) \cdot \exp \left\{\frac{1}{N} \exp \left\{-F\left(\gamma^{(0)}\right)\right\}\right. \\
\left.\cdot\left|W\left(\gamma_{i}^{A} ; D, c\right)\right|\right\} \leqq R_{3} \exp \left\{-2 F\left(\gamma^{(0)}\right)\right\}
\end{gathered}
$$

for a constant $R_{3}=R_{3}(D, c)$. We have

$$
\Sigma^{(r, m)}=\sum_{J} \Sigma^{(J, m)},
$$

where the sum $\Sigma^{(J, m)}$ has the same form as $\Sigma^{(r, m)}$ with the additional condition of fixing the indices $j \in J$ for which $\hat{\gamma}_{j}^{A}$ are the least contours. It corresponds to our general strategy to consider separately the contribution of least contours.

For $\Sigma^{(J, m)}$ we can write the estimate

$$
\begin{aligned}
& \Sigma^{(J, m)} \leqq\left(R_{3} \exp \left\{-2 F\left(\gamma^{(0)}\right)\right\}\right)^{|J|} \sum_{\gamma_{i}^{A} \in T\left(z_{i}\right), i \in I=\{1, \ldots, r)-J}
\end{aligned}
$$

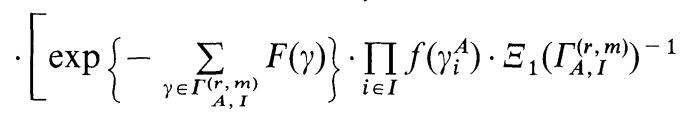

$$
\begin{aligned}
& \left.\cdot \exp \left\{\frac{1}{N} \cdot \exp \left\{-F\left(\gamma^{(0)}\right)\right\} \cdot\left|W\left(\Gamma_{A, I}^{(r, m)} ; D, c\right)\right|\right\}\right] \\
& =\left(R_{3} \exp \left\{-2 F\left(\gamma^{(0)}\right)\right\}\right)^{|J|} . \sum_{\substack{\gamma_{i}^{A} \in T\left(z_{i}\right), i \in I \\
\gamma_{q}^{\prime} \in T\left(z_{q}^{\prime}\right), 1 \leqq q \leqq m}}
\end{aligned}
$$

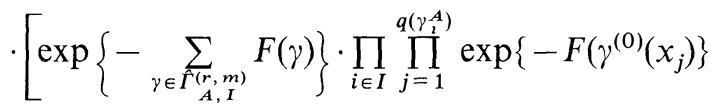

$$
\begin{aligned}
& \left.\cdot\left(\Xi_{1}\left(\Gamma_{A, I}^{(r, m)}\right)\right)^{-1} \cdot \exp \left\{\frac{1}{N} \exp \left\{-F\left(\gamma^{(0)}\right)\right\}\left|W\left(\Gamma_{A, I}^{(r, m)} ; D, c\right)\right|\right\}\right] \text {. }
\end{aligned}
$$


It is not difficult to see that

$$
\prod_{i \in I} \prod_{j=1}^{q\left(\gamma_{i}^{A}\right)} \exp \left\{-F\left(\gamma_{x_{j}}^{(0)}\right)\right\} \leqq \exp \left\{\frac{1}{N} \exp \left\{-F\left(\gamma^{(0)}\right)\right\} \cdot\left|\tilde{W}\left(\hat{\Gamma}_{A, I}^{(r)}\right)\right|,\right.
$$

where

$$
\tilde{W}\left(\hat{\Gamma}_{A, I}^{(r)}\right)=\bigcup_{i \in I}\left(V\left(\tilde{b_{i}}\right) \backslash \tilde{b_{i}}(\mathrm{int})\right) \cap \mathrm{W}_{3}\left(\Gamma_{p}\right), \quad \tilde{b_{i}}=b_{i}^{A} \backslash S .
$$

Consequently

$$
\begin{aligned}
\Sigma^{(J, m)} \leqq & \left(R_{3} \exp \left\{-2 F\left(\gamma^{(0)}\right)\right\}\right)^{|J|} \sum_{\substack{\gamma A \\
\gamma_{i}^{A} \mid d\left(z_{1}, \gamma_{1}^{A}\right) \leqq r_{1} \\
\gamma_{q}^{\prime} \in T\left(z_{q}^{\prime}\right), \gamma_{q}^{\prime} \neq \gamma^{(0)}}} \\
& \cdot \exp \left\{-\sum_{\gamma \in \Gamma_{A, I}^{(r, m)}} F(\gamma)\right\} \cdot\left(\Xi_{1}\left(\hat{\Gamma}_{A, I}^{(r, m)}\right)\right)^{-1} \\
& \cdot \exp \left\{\frac{1}{N} \exp \left\{-F\left(\gamma^{(0)}\right)\right\} \cdot\left[\left|\tilde{W}\left(\hat{\Gamma}_{A, I}^{(r)}\right)\right|+W\left(\Gamma_{A, I}^{(r, m)} ; D, c\right)\right]\right\} .
\end{aligned}
$$

This sum can be estimated as follows. First we can sum up over all contours $\gamma_{\ell}^{\prime}$, $\ell=1, \ldots, m$ corresponding to some smooth contours $\bar{\gamma}_{\ell}^{\prime}, \ell=1, \ldots, m$ and over all contours $\hat{\gamma}_{i}^{A}, i \in I$, which correspond to some smooth contours $\bar{\gamma}_{i}^{A}, i \in I$, and not intersect $\partial W_{1}\left(\Gamma_{A, I}^{(r, m)}\right.$ ) (here we use the property 3 ) of contour functional $F$ with the following choice of the set $W: W=V\left(\bar{\gamma}_{\ell}^{\prime}\right)$ for contours $\bar{\gamma}_{\ell}^{\prime}, 1 \leqq \ell \leqq m$ and $W=V\left(\bar{\gamma}_{i}^{A}\right) \backslash \tilde{W}\left(\hat{\Gamma}_{A, I}^{(r)}\right)$ for contours $\left.\bar{\gamma}_{i}^{A}, i \in I\right)$; then we estimate the sum over smooth contours using Lemma 2.

The result of the first summation gives us the estimate:

$$
\begin{aligned}
& \Sigma^{(J, m)} \leqq\left(R_{3} \exp \left\{-2 F\left(\gamma^{(0)}\right)\right\}\right)^{|J|}\left(C\left(r_{1}\right)\right)^{|I|+m}
\end{aligned}
$$

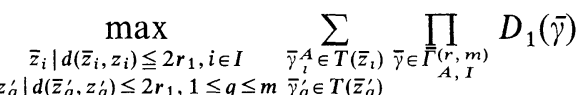

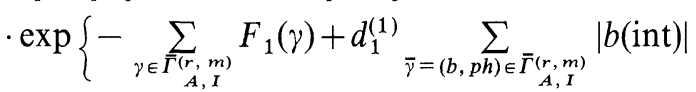

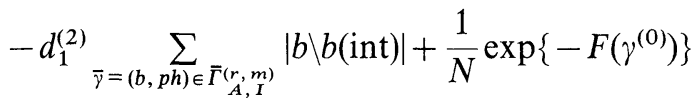

$$
\begin{aligned}
& \cdot\left(\sum_{\bar{\gamma}=(b, p h) \in \bar{\Gamma}_{A, I}^{(r, m)}}|V(b) \backslash b(\mathrm{int})|+D_{2} \Sigma\left|\partial\left(s \gamma^{(\mathrm{ver})}\right)\right|\right) \cdot\left(\Xi_{1}\left(\bar{\Gamma}_{A, I}^{(r, m)}\right)\right)^{-1} .
\end{aligned}
$$

Here $C\left(r_{1}\right)$ is a constant, $D_{1}(\bar{\gamma})=\max _{b_{1} \subset b}\left|\partial b_{i}\right|, \bar{\gamma}=(b, p h), b_{i}$ is the connected component of $b$. It is clear that $\left|D_{1}(\bar{\gamma})\right|<\max _{i}\left|\partial b_{i}^{(\text {ver })}\right|^{2}, D_{2}$ is a constant.

For any contour $\gamma=(b, p h)$ we denote by $n(\gamma)$ the number of connected components of the set $b$ which differ from the least components. If $b=U b_{i}$ is a decomposition on connected components, then we can assume that $b_{i} \neq b^{(0)}$ for $i \leqq n(\gamma)$ and $b_{i}=b^{(0)}$ for $i>n(\gamma)$.

Let $\mathscr{M}\left(\bar{b}_{1}, \ldots, \bar{b}_{n}\right)$ be the set of all contours $\gamma=(b, p h)$ for which $n(\gamma)=n$ and let $b_{i}$ $=\bar{b}_{i}, 1 \leqq i \leqq n(\gamma), \mathscr{M}(\emptyset)$ be the set of contours with $n(\gamma)=0$. 
Consider separately the sum over such configurations $\bar{\Gamma}_{A, I}^{(r, m)}$ for which

$$
\bar{\gamma}_{i}^{A} \in \mathscr{M}\left(b_{1}^{(i)}, \ldots, b_{n_{l}}^{(i)}\right), \quad \bar{\gamma}_{q}^{\prime} \in \mathscr{M}\left(b_{1}^{(q)}, \ldots, b_{n_{q}^{\prime}}^{(q)}\right),
$$

$i \in I, 1 \leqq q \leqq m$. This sum is not more than

$$
\begin{aligned}
& \exp \left\{-\sum_{\gamma \in \bar{\Gamma}_{A, I}^{(,, m)}}\left[\left(K_{1}-D_{3}\right) \cdot \sum_{j=1}^{n_{i}}\left|\left(\partial b_{j}^{(i)}\right)^{(\mathrm{ver})}\right|-d_{1}^{(1)} \sum_{j=1}^{n_{i}}\left|b_{j}^{(i)}(\mathrm{int})\right|\right.\right. \\
& \left.\left.-d_{1}^{(2)} \sum_{j=1}^{n_{i}}\left|b_{j}^{(i)} \backslash b_{j}^{(i)}(\mathrm{int})\right|\right]\right\} \cdot \exp \left\{-\sum_{q=1}^{m}\left[\left(K_{1}-D_{3}\right)\right.\right. \\
& \left.\left.\cdot \sum_{\ell=1}^{n_{q}^{\prime}}\left|\left(\partial b_{q}^{(\ell)}\right)^{(\mathrm{ver})}\right|-d_{1}^{(1)} \sum_{\ell=1}^{n_{q}^{\prime}}\left|b_{q}^{(\ell)}(\mathrm{int})\right|-d_{1}^{(2)} \sum_{\ell=1}^{n_{q}^{\prime}}\left|b_{q}^{(\ell)} \backslash b_{q}^{(\ell)}(\mathrm{int})\right|\right]\right\} \\
& \left.\cdot \prod_{i \in I}\left(R_{4} \exp \left\{-F\left(\gamma^{(0)}\right)\right\}\right)^{d\left(b_{1}^{(1)}, \ldots, b_{n_{1}}^{(1)}\right)} \cdot \prod_{j=1}^{m}\left(R_{4} \exp \left\{-F\left(\gamma^{(0)}\right)\right\}\right)^{d\left(b_{1}^{(j)}, \ldots, b_{n_{i}}^{(j)}\right)}\right) \\
& \cdot \exp \left\{-\frac{1}{N} \exp \left\{-F\left(\gamma^{(0)}\right)\right\} \cdot(1-\varrho) \cdot\left|W_{0}\left(\bar{\Gamma}_{A, I}^{(r, m)}\right)\right|\right\}
\end{aligned}
$$

where

$$
W_{0}\left(\bar{\Gamma}_{A, I}^{(r, m)}\right)=\left(\bigcup_{i \in I} \bigcup_{j=1}^{n_{i}} b_{j}^{(i)}\right) \cup\left(\bigcup_{i=1}^{m} \bigcup_{j=1}^{n_{j}^{\prime}} V\left(b_{j}^{(i)}\right)\right), \quad d\left(b_{1}, \ldots, b_{n}\right)
$$

is the length of the minimal tree which connects the sets $W_{3}\left(b_{i}\right), 1 \leqq i \leqq n, W_{3}\left(b_{i}\right)$ $=W\left(\gamma_{i}\right), \gamma_{i}=\left(b_{i}, p h\right), \varrho \leqq \exp \left\{-C_{1} K_{1}\right\}, D_{3}, R_{4}, C_{2}$ are absolute constants. Now we see that the last sum is not more than

$$
\begin{aligned}
& \prod_{\gamma_{1} \in \bar{\Gamma}_{A, I}^{(r, m)} \mid \eta_{i} \in \mathcal{M}\left(b_{1}^{(i)}, \ldots, b_{t_{1}}^{(i)}\right)} \\
& \cdot\left[\exp \left\{-\left(K_{1}-D_{3}\right) \sum_{j=1}^{t_{2}}\left|\left(\partial b_{j}^{(i)}\right)^{(\mathrm{ver})}\right|-d_{2} \sum_{j=1}^{t_{2}}\left|b_{j}^{(i)}\right|\right\}\right. \\
& \left.\cdot\left(R_{4} \exp \left\{-F\left(\gamma^{(0)}\right)\right\}\right)^{d\left(b_{1}^{(i)}, \ldots, b_{t_{l}}^{(i)}\right)}\right] \\
& \cdot \prod_{\gamma \in \bar{\Gamma}_{A, I}^{(r, m)} \mid \gamma \in \mathcal{M}(\emptyset)}\left(R_{5} \exp \left\{-2 F\left(\gamma^{(0)}\right)\right\}\right) .
\end{aligned}
$$

Here

$$
d_{2}=\min \left(\left(\exp \left\{-F\left(\gamma^{(0)}\right)\right\}-d_{1}^{(1)}\right)(1-\varrho), d_{1}^{(2)}\left(r_{2}\right)^{-1}\right),
$$

$R_{5}$ is an absolute constant, $r_{2}=9$ if $p h=$ f.g.s. and $r_{2}=6$, if $p h=(3.3)$-g.s.

Now Lemma 3 follows from Lemma 2.

From the main estimate of Lemma 3 it is not difficult to see that the operator $\Lambda$ is a contraction and $\|\Lambda\| \leqq \operatorname{const}\left(K_{1}\right) \rightarrow 0$ as $K_{1} \rightarrow \infty$. Therefore for sufficiently large $K_{1}$ the limit correlation functions can be written as $\pi=\sum_{i=0}^{\infty} \Lambda^{i} \Phi$. The estimation of the difference between the limit correlation functions and the correlation functions in a finite volume is done by a routine method (see $[5,6])$. 


\section{Proof the Theorem B}

We shall consider the case of contours $\gamma^{(f)}$ and $\Xi_{W}^{(s)}\left(\gamma^{(f)} \mid \beta, \delta\right)$. Other cases can be considered in the same manner. We have (see Sect. 4).

$$
\begin{aligned}
& \Xi_{W}^{(s)}\left(\gamma^{(f)} \mid \beta, \delta\right) \\
& =\sum_{\substack{\gamma_{1}=\left(b_{1}, p h\right) \in S m\left(\gamma^{(f)}\right) \\
b_{i}(\mathrm{int}) \in W}} \exp \left\{\beta \mathscr{H}^{(f)}\left(b_{1}^{(f)}\right)\right\} \cdot \sum_{\varphi\left(b_{1}\right) \in \mathfrak{U}\left(\gamma_{1}^{(f)}\right)} \\
& \cdot \exp \left\{\beta \left[\sum _ { x \in b _ { 1 } } \varphi ( x ) \left(\frac{1}{2} J_{0} \sum_{\left(x, x^{\prime}\right) \in\left(\mathbb{Z}^{3}\right)_{\mathrm{hor}}^{(1)}} \varphi\left(x^{\prime}\right)+\frac{1}{2} J_{1} \sum_{\left(x, x^{\prime}\right) \in\left(\mathbb{Z}^{3}\right)_{\mathrm{ver}}^{(1)}} \varphi\left(x^{\prime}\right)\right.\right.\right. \\
& \left.\left.\left.-\frac{1}{2} J_{2} \sum_{\left(x, x^{\prime}\right) \in\left(\mathbb{Z}^{3}\right)_{\text {ver }}^{(2)}} \varphi\left(x^{\prime}\right)\right)\right]\right\} \text {. }
\end{aligned}
$$

Here $\mathscr{H}^{(f)}(b)$ is the energy of the ferromagnetic configuration in $b$.

For any $\varphi(b)=\varphi$ we denote

$\left.\mathrm{a}_{1}\right) G^{(\mathrm{ver})}(\varphi)$ is the set of ordered bonds $\left(x, x^{\prime}\right) \in\left(\mathbb{Z}^{3}\right)_{\text {hor }}^{(1)}, x \in b$, for which $\varphi(x)$ $\neq \varphi\left(x^{\prime}\right)$ and $d\left(\left(x, x^{\prime}\right),(\partial(s b))^{\text {ver }}\right) \leqq r_{1}$. We recall that $\partial(s b)^{(\text {ver })}$ is the set of vertical faces of $\partial(s b)$ which can be also considered as the set of horizontal bonds perpendicular to these faces.

$\left.\mathrm{a}_{2}\right) G_{1}^{(\mathrm{ver})}(\varphi)$ is the set of ordered bonds $\left(x, x^{\prime}\right) \in\left(\mathbb{Z}^{3}\right)_{\text {hor }}^{(1)}$, where $x \in b, \varphi(x)$ $\neq \varphi\left(x^{\prime}\right)$ and $\left(x, x^{\prime}\right) \notin G^{(\mathrm{ver})}(\varphi)$.

$\left.\mathrm{a}_{3}\right) G_{2}^{(\mathrm{ver})}(\varphi)$ is the set of $x \in b$ for which $d\left(x, G^{\text {(ver) }}(\varphi) \cup G_{1}^{\text {(ver) }}(\varphi)\right) \leqq 2 r_{1}$. The values of $\varphi(x), x \in G_{2}(\varphi)$, are defined uniquely by values of $\varphi$ on $G^{(\text {ver })}(\varphi)$ $\cup G_{1}^{\text {(ver) }}(\varphi)$.

$\left.\mathrm{a}_{4}\right) W_{1}(\varphi)=b \backslash G_{2}^{(\mathrm{ver})}(\varphi)$.

$\left.\mathrm{a}_{5}\right) N_{1}(\varphi)$ is the set of $x=\left(x_{1}, x_{2}, x_{3}\right) \in W_{1}(b)$, where $\varphi\left(x_{1}, x_{2}, x_{3}+1\right)=\varphi\left(x_{1}\right.$, $\left.x_{2}, x_{3}-1\right) \neq \varphi\left(x_{1}, x_{2}, x_{3}\right)$.

$\left.\mathrm{a}_{6}\right) N_{r}(\varphi), r>1$ is the number of series of points lying on the same vertical line, i.e. series of points $\left(x_{1}, x_{2}, x^{\prime}\right) \in W_{1}(b), a<x^{\prime} \leqq a+r$, and $\varphi\left(x_{1}, x_{2}, a\right) \neq \varphi\left(x_{1}, x_{2}\right.$, $a+1)=\varphi\left(x_{1}, x_{2}, a+2\right)=\ldots=\varphi\left(x_{1}, x_{2}, a+r\right) \neq \varphi\left(x_{1}, x_{2}, a+r+1\right), N_{r}^{1}(\varphi)$ is the set of points of the form $\left(x_{1}, x_{2}, a+1\right)$ or $\left(x_{1}, x_{2}, a+r\right), N_{r}^{>2}(\varphi)$ is the set of points of the form $\left(x_{1}, x_{2}, a+\ell\right), 2<\ell<r-1, N_{r}^{2}(\varphi)$ is the set of points of the form $\left(x_{1}\right.$, $\left.x_{2}, a+2\right),\left(x_{1}, x_{2}, a+r-1\right)$. An important remark is that $r \leqq 2 r_{1}$, because otherwise there will be points in a ferromagnetic phase.

We rewrite the energy of $\varphi(b)$ as follows:

$$
\begin{aligned}
-H(\varphi(b))= & -\mathscr{H}^{(f)}(b)-\frac{1}{2} J_{0}\left|G^{(\mathrm{ver})}(\varphi)\right|-\frac{1}{2} J_{0}\left|G_{1}^{(\mathrm{ver})}(\varphi)\right| \\
& -2 \delta \sum_{r \geqq 2} N_{r}(\varphi)-4 J_{1} N_{1}(\varphi)+\mathscr{H}_{1} .
\end{aligned}
$$

Here $\mathscr{H}_{1}$ is a correction term $\left|\mathscr{H}_{1}\right| \leqq$ const $\cdot\left(J_{1}+J_{2}\right) \cdot\left(\left|G_{1}(\varphi)\right|+\left|G_{2}(\varphi)\right|\right)$. We recall our assumption that $J_{1}, J_{2}$ are sufficiently small comparing with $J_{0}$.

Lemma 1. There exists a constant $c_{2}$ such that

$$
\left|G^{(\mathrm{ver})}\right|>c_{2}\left|\partial(s b)^{(\mathrm{ver})}\right| .
$$

Proof. Let a bond $\left(x, x^{\prime}\right)$ be orthogonal to a face of $\partial(s b)^{(v e r)}$ and $\varphi(x)$ is in a phase while $x^{\prime} \in \partial(s b)^{(\mathrm{ver})}$ and $\varphi\left(x^{\prime}\right)$ is not in the phace. It means that in the neighbourhood 
of the radius $r_{1}$ there must be horizontal bond $\left(y, y^{\prime}\right) \subset b$, where $\varphi(y) \neq \varphi\left(y^{\prime}\right)$. Otherwise $\varphi\left(x^{\prime}\right)$ will be in a phase. Q.E.D.

The cardinality of the set of connected components $\partial b_{i}^{(\mathrm{ver})},\left|\partial b_{i}^{(\mathrm{ver})}\right|=n$, passing through a fixed face is not more than $c_{3}^{n}$, where $c_{3}$ is a constant. We can choose $c_{3}$ so large that the cardinality of the set of all possible $\varphi\left(G^{(\mathrm{ver})}\right)$ is not more than

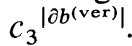

A point $x=\left(x_{1}, x_{2}, x_{3}\right) \in b \backslash G^{(\mathrm{ver})}(\varphi)$ is called an elementary defect (e.d.) if $\varphi(x)$ $\neq \varphi(y)$ for all $y=\left(y_{1}, y_{2}, y_{3}\right),\left|y_{1}-x_{1}\right|+\left|y_{2}-x_{2}\right|=1, y_{3}=x_{3}$, and there are no other horizontal bonds $\left(x^{\prime}, x^{\prime \prime}\right) \in b$ such that $\left(x^{\prime}, x^{\prime \prime}\right) \cap b^{(0)}(x) \neq \emptyset$ and $\varphi\left(x^{\prime}\right) \neq \varphi\left(x^{\prime \prime}\right)$.

A configuration $\varphi(b)$ is called ideal if there are no e.d. The set of ideal configurations in $b$ is denoted by $\mathfrak{2}^{(i)}(b)$.

Let $\varphi_{0}(b) \in \mathfrak{O}^{(i)}(b)$. The set of all $\varphi(b)$ which differ from $\varphi_{0}(b)$ by some number of e.d. is denoted by $\mathfrak{H}\left(b \mid \varphi_{0}(b)\right)$. Each $\varphi(b)$ corresponds for one and only one ideal configuration $\varphi_{0}(b)$.

We can write

$$
\begin{aligned}
& \Xi_{W}^{(s)}\left(\gamma^{(f)} \mid \beta, \delta\right) \\
& =\sum_{\substack{\gamma_{1}^{(f)}=\left(b^{(f)}, p h\right) \in S m \\
b_{1}^{(f)}(\mathrm{int}) \subset W}} \sum_{\varphi(f)} \sum_{\varphi\left(b_{1}^{(f)}\right) \in \mathfrak{Q}\left(\gamma_{1}^{(f)}\right)} \\
& \cdot \exp \left\{-\beta\left[\frac{1}{2} J_{0}\left|G^{(\mathrm{ver})}\left(\varphi\left(b_{1}^{(f)}\right)\right)\right|+\frac{1}{2} J_{0}\left|G_{1}^{(\mathrm{ver})}\left(\varphi\left(b_{1}^{(f)}\right)\right)\right|\right.\right. \\
& \left.\left.+2 \delta \sum_{r=2}^{2 r_{1}}\left|N_{r}\left(\varphi\left(b_{1}^{(f)}\right)\right)\right|+4 J_{1} N_{1}\left(\varphi\left(b_{1}^{(f)}\right)\right)+\mathscr{H}_{1}\left(\varphi\left(b_{1}^{(f)}\right)\right)\right]\right\} \\
& =\sum_{\substack{\gamma_{1}^{(f)}=\left(b^{(f)}, p h\right) \in S m \gamma^{(f)} \\
b_{1}^{(f)}(\mathrm{int}) \subset W}} \sum_{\varphi\left(b_{1}^{(f)}\right) \in \mathfrak{Q}\left(\gamma_{1}^{(f)}\right)} \\
& \cdot \exp \left\{-\beta\left[\frac{1}{2} J_{0}\left|G^{(\mathrm{ver})}\left(\varphi\left(b_{1}^{(f)}\right)\right)\right|+\frac{1}{2} J_{0}\left|G_{1}^{(\mathrm{ver})}\left(\varphi\left(b_{1}^{(f)}\right)\right)\right|\right.\right. \\
& \left.\left.+\delta \sum_{r=2}^{2 r_{1}}\left|N_{r}^{(1)}\left(\varphi\left(b_{1}^{(f)}\right)\right)\right|+4 J_{1} N_{1}\left(\varphi\left(b_{1}^{(f)}\right)\right)+\mathscr{H}_{1}\left(\varphi\left(b_{1}^{(f)}\right)\right)\right]\right\} \text {. }
\end{aligned}
$$

Furthermore

$$
\begin{aligned}
& \Xi_{W}^{(s)}\left(\gamma^{(f)} \mid \beta, \delta\right)
\end{aligned}
$$

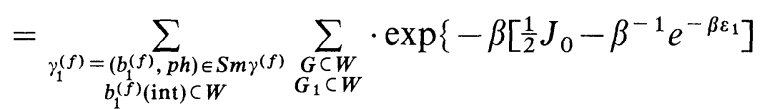

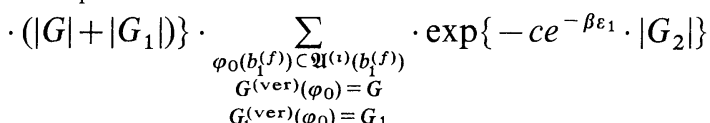

$$
\begin{aligned}
& \cdot \exp \left\{-\beta\left[4 J_{1} N_{1}\left(\varphi_{0}\left(b_{1}^{(f)}\right)\right)+\delta \sum_{r=2}^{2 r_{1}}\left|N_{r}^{(1)}\left(\varphi_{0}\left(b_{1}^{(f)}\right)\right)\right|+\mathscr{H}_{1}\right]\right\} \\
& \sum_{\left.\varphi\left(b_{1}^{(f)}\right) \in \mathfrak{U}\left(b_{1}^{(f)}\right) \mid \varphi_{0}\left(b_{1}^{(f)}\right)\right)} \cdot \exp \left\{-\beta \sum_{n=0}^{4} n_{i}\left(\varphi\left(b_{1}^{(f)}\right) \cdot \varepsilon_{i}\right\} .\right.
\end{aligned}
$$


Here $n_{i}\left(\varphi\left(b_{1}^{(f)}\right)\right)$ is the number of e.d. of type $i$ of configuration $\varphi\left(b_{1}^{(f)}\right)$ (see Sect. 1) and $c$ is an absolute constant,

$$
0<c<\min _{G, G_{1}}\left(\left|G_{2}\right|^{-1}\left(|G|+\left|G_{1}\right|\right) .\right.
$$

Let $v_{i}\left(\varphi_{0}\left(b_{1}^{(f)}\right)\right)$ be the set of points $x \in b_{1}^{(f)}($ int $) \backslash G_{2}$, where the spin-flip changes the energy $\varepsilon_{i}$.

Then

$$
\begin{aligned}
\Sigma & \exp \left\{-\beta \sum_{i=0}^{4} n_{i} \varepsilon_{i}\right\} \leqq \sum_{\left\{n_{i}\right\}} \prod_{i=0}^{4} C_{\left|v_{i}\right|}^{n_{i}} e^{-\beta \varepsilon_{i} n_{i}} \\
& \approx \exp \left[\sum_{i=0}^{4}\left|v_{i}\right| \ln \left(1+\exp \left(-\beta \varepsilon_{i}\right)\right)\right] \\
& \approx \exp \left(\left|v_{0}\right| \exp \left(-\beta \varepsilon_{0}\right)+\left(\left|v_{1}\right|+\left|v_{2}\right|\right) \exp \left(-\beta \varepsilon_{1}\right)\right) .
\end{aligned}
$$

It is easy to see that

$$
\begin{gathered}
\left|v_{0}\left(\varphi_{0}\right)\right|=\sum_{r \geqq 3}\left|N_{r}^{(1)}\left(\varphi_{0}\right)\right|, \quad\left|v_{1}\left(\varphi_{0}\right)\right|=\sum_{r \geqq 4}^{2 r_{1}}\left|N_{r}^{>2}\left(\varphi_{0}\right)\right|, \\
\left|v_{2}\left(\varphi_{0}\right)\right|=\left|N_{2}^{(1)}\left(\varphi_{0}\right)\right| .
\end{gathered}
$$

We remark that every configuration $\varphi_{0}\left(b_{1}^{(f)}\right)$ can be uniquely extended to $V(b) \backslash G \cup G_{1}$ and different configurations will have different extensions. As a result we can write

$$
\begin{aligned}
& \Xi_{W}^{(s)}\left(\gamma^{(f)} \mid \beta, \delta\right) \\
& \leqq \sum_{\substack{G \subset W \\
G_{1} \subset W}} \exp \left\{-\left(\frac{1}{2} \beta J_{0}-e^{-\beta \varepsilon_{1}}\right)\left(|G|+\left|G_{1}\right|\right)\right\} \cdot \sum_{\substack{\varphi_{0}(V(b)) \\
G(\operatorname{ver})\left(b_{0}\right)=G \\
G_{1}^{(v e r)}\left(\varphi_{0}\right)=G_{1}}} \\
& \cdot \exp \left\{-c e^{-\beta \varepsilon_{1}}\left|G_{2}\right|\right\} \cdot \exp \left\{-\beta\left[4 J_{1} \mid N_{1}\left(\varphi_{0}(V(b) \cap W) \mid\right.\right.\right. \\
& +\delta \sum_{r=2}^{2 r_{1}}\left|N_{r}^{(1)}\left(\varphi_{0}(V(b))\right) \cap b\right|-\beta^{-1} e^{-\beta \varepsilon_{0}} \sum_{r \geqq 3} \mid N_{r}^{(1)}\left(\varphi_{0}(V(b)) \cap W \mid\right. \\
& -\beta^{-1} e^{-\beta \varepsilon_{1}} \sum_{r=5}^{2 r_{1}}\left|N_{r}^{>2}\left(\varphi_{0}(V(b))\right) \cap W\right|-\beta^{-1} e^{-\beta \varepsilon_{1}} \\
& \left.\left.\cdot\left|N_{2}^{(1)}(\varphi(V(b))) \cap W\right|+\text { v.s.n. }\left|W-G_{2}\right|+\mathscr{H}_{1}\right]\right\} \text {. }
\end{aligned}
$$

Remember that $2 \beta \delta-2 e^{-\beta \varepsilon_{0}}+3 e^{-\beta \varepsilon_{1}}$ is v.s.n.. We have

$$
\begin{aligned}
\beta \delta & \sum_{r=2}^{2 r_{1}}\left|N_{r}^{(1)}\left(\varphi_{0}(V(b))\right) \cap b\right| \\
& -e^{-\beta \varepsilon_{0}} \sum_{r \geqq 3}\left|N_{r}^{(1)}\left(\varphi_{0}(V(b))\right) \cap b\right|-e^{-\beta \varepsilon_{1}} \sum_{r=5}^{2 r_{1}}\left|N_{r}^{>2}\left(\varphi_{0}(V(b))\right) \cap b\right| \\
& -e^{-\beta \varepsilon_{1}}\left|N_{2}^{(1)}\left(\varphi_{0}(V(b))\right) \cap b\right|=\left(\beta \delta-2 e^{-\beta \varepsilon_{1}}\right)\left|N_{2}^{(1)}\left(\varphi_{0}(V(b))\right) \cap b\right| \\
& +\frac{1}{2} e^{-\beta \varepsilon_{1}} \sum_{r=3}^{2 r_{1}}\left|N_{r}^{(1)}\left(\varphi_{0}(V(b))\right) \cap b\right| \\
& -e^{-\beta \varepsilon_{1}}\left|b \backslash G_{2}\right|+e^{-\beta \varepsilon_{1}} \sum_{r>2}\left|N_{r}^{(2)}\left(\varphi_{0}(V(b))\right) \cap b\right| .
\end{aligned}
$$


As $\gamma=(b, p h)$ is the contour we have

$$
\begin{gathered}
\left.\frac{1}{2} e^{-\beta \varepsilon_{1}} \sum_{r=3}^{2 r_{1}} \mid N_{r}^{(1)}\left(\varphi_{0}(V(b))\right) \cap b(\text { int })\right) \mid+\left(\beta \delta-2 e^{-\beta \varepsilon_{1}}\right) \\
\cdot \mid N_{2}^{(1)}\left(\varphi_{0}(V(b))\right) \cap b(\text { int })\left|>d e^{-\beta \varepsilon_{1}}\right| b(\text { int }) \backslash G_{2} \mid
\end{gathered}
$$

and

$$
\mid N_{r}^{(2)}\left(\varphi_{0}(V(b))\right) \cap\left(b \backslash b(\text { int }) \cup G_{2}\right)\left|>d_{1}\right| b \backslash b(\text { int }) \cup G_{2} \mid,
$$

where $d, d_{1}$ are absolute constants. Also

$$
\begin{aligned}
e^{-\beta \varepsilon_{1}} & \left.\mid N_{r}^{(1)}\left(\varphi_{0}(V(b))\right) \cap(V(b) \backslash b) \cap W\right) \mid \\
& \left.+e^{-\beta \varepsilon_{1}} \mid N_{r}^{>2}\left(\varphi_{0}(V(b))\right) \cap(V(b) \backslash b) \cap W\right) \mid \\
\leqq & e^{-\beta \varepsilon_{1}}|(V(b) \backslash b) \cap W| .
\end{aligned}
$$

If the sets $G$ and $G_{1}$ are fixed, then the number of ideal configurations $\varphi_{0}(V(b))$ is not more than const ${ }^{|G|+\left|G_{1}\right|}$.

Thus

$$
\begin{aligned}
& \Xi_{W}^{(s)}\left(\gamma^{(f)} \mid \beta, \delta\right) \leqq \sum_{\substack{G_{1} \subset W \\
G \subset W}} \exp \left\{-\left(\beta J_{0}-\text { const }\right)\left(|G|+\left|G_{1}\right|\right)\right\} \\
& \cdot \exp \left\{e^{-\beta \varepsilon_{1}}\left|V(b) \cap W \backslash\left(b \cup G \cup G_{1}\right)\right|\right. \\
& \left.+\bar{d} e^{-\beta \varepsilon_{1}} \mid b(\text { int }) \backslash\left(G \cup G_{1}\right)\left|-\bar{d}_{1} e^{-\beta \varepsilon_{1}}\right| b \backslash b(\text { int }) \mid\right\},
\end{aligned}
$$

where $d, \bar{d}_{1}$ are positive constants and $0<\bar{d}<1$.

Lemma 2. Put $e^{-G_{1}^{(f)}(\gamma(f))}=\sum_{G} \exp \left\{-\left(\frac{\beta}{2} J_{0}-\right.\right.$ const $\left.)|G|\right\}$. Then

$$
\begin{aligned}
& e^{-G_{1}^{(f)}(\gamma(f)} \cdot \sum_{G, G_{1}} \exp \left\{-\left(\frac{\beta}{2} J_{0}-\text { const }\right)\left(|\mathrm{G}|+\left|\mathrm{G}_{1}\right|\right)\right\} \\
& \leqq \exp \{\text { v.s.n. }|V(b)|\} .
\end{aligned}
$$

We shall give only the sketch of the proof. We decompose $G \cup G_{1}$ onto connected components and get a usual contour model (see [6]). We remark that the statistical weights of the least components are v.s.n. because we excluded e.d. The needed estimate follows by a direct application of the method of correlation equations (see [6]).

Thus we get the desired result.

\section{Solution of (5) and (6) and Final Remarks}

Now we return to Eqs. (5), (6). We look for the solution $F^{(f)}=F_{1}^{(f)}+F_{2}^{(f)}, F^{(3,3)}$ $=F_{1}^{(3,3)}+F_{2}^{(3,3)}$. From the very beginning we put $F_{1}^{(f)}\left(\gamma^{(f)}\right)=G_{1}^{(f)}\left(\gamma^{(f)}\right), F_{1}^{(3,3)}\left(\gamma^{(f)}\right)$ $=G_{1}^{(3,3)}\left(\gamma^{(3,3)}\right)$. Assumption 1 follows easily from Theorem B. Equations (5), (6) for $F_{2}$ can be rewritten in the following form: 


$$
\begin{aligned}
& G_{2}^{(f)}\left(\gamma^{(f)}\right)=F_{2}^{(f)}\left(\gamma^{(f)}\right)-\Delta^{(c r)}\left(\gamma^{(f)} \mid F^{(f)}\right)+\sum_{s} \Delta\left(O_{s} \mid F^{\left(\kappa_{s}\right)}\right), \\
& G_{2}^{(3,3)}\left(\gamma^{(3,3)}\right)= F_{2}^{(3,3)}\left(\gamma^{(3,3)}\right)-\beta\left(h^{(f)}-h^{(3,3)}\right) \cdot|b| \\
&-\Delta^{(c r)}\left(\gamma^{(3,3)} \mid F^{(3,3)}\right)+\sum_{s} \Delta\left(O_{s} \mid F^{\left(\kappa_{s}\right)}\right) .
\end{aligned}
$$

Let us put

$$
G_{3}^{(3,3)}\left(\gamma^{(3,3)}\right)=G_{2}^{(3,3)}\left(\gamma^{(3,3)}\right)+\beta\left(h^{(f)}-h^{(3,3)}\right) \cdot|b| .
$$

Then (17) means that

$$
\left(G_{2}^{(f)}, G_{3}^{(3,3)}\right)=\left(F_{2}^{(f)}, F_{2}^{(3,3)}\right)+T\left(F_{2}^{(f)}, F_{2}^{(3,3)}\right) .
$$

It follows from Theorem B that $G_{2}^{(f)} \geqq-d_{2}^{(1)} \exp \left\{-\beta \varepsilon_{1}\right\} \cdot|b|, d_{2}^{(1)}$ is some absolute positive constant independent of $\beta, \bar{d}_{2}^{(1)}<1$. For $G_{3}^{(3,3)}$ we have

$$
G_{3}^{(3,3)} \geqq\left[-\frac{2}{3} e^{-\beta \varepsilon_{0}}+\left(1-d_{2}^{(1)}\right) e^{-\beta \varepsilon_{1}}\right] \cdot|b|,
$$

with the same $d_{2}^{(1)}$. From the other side

$$
\sum_{\substack{\gamma \in T_{0}(z) \\ \gamma \neq \gamma^{(0)}}} \exp \left\{-F_{1}\left(\partial b^{(\mathrm{ver})}\right)\right\}
$$

is v.s.n. In the case of $f$-phase $N=1$ and $\exp \left\{-F\left(\gamma^{(0)}\right)\right\}=e^{-\beta \varepsilon_{1}}$, in (3,3)-phase, $N=6$ and

$$
\frac{1}{N} \exp \left\{-F\left(\gamma^{(0)}\right)\right\}=\frac{2}{3} \exp \left\{-\beta \varepsilon_{0}\right\}+\text { v.s.n. }
$$

Thus the conditions of Theorem $\mathrm{A}$ are valid for sufficiently large $\beta$.

It follows from the proof of Theorem B that if a contour consists of several components then the values of $G_{2}^{(f)}, G_{2}^{(3,3)}$ are sums of values of different components, i.e. Assumption 4 for contour models is true.

In the space of pairs $\left(F_{2}^{(f)}, F_{2}^{(3,3)}\right)$ we introduce the norm

$$
\left\|\left(F_{2}^{(f)}, F_{2}^{(3,3)}\right)\right\|=\max \left(\left\|F_{2}^{(f)}\right\|,\left\|F_{2}^{(3,3)}\right\|\right) .
$$

Then in (18) the operator $T$ is a contraction and the contraction coefficient is a v.s.n. as one can easily check. Thus we get the solution of (5), (6). It will satisfy also Assumptions 4, Sect. 3 because $G$ satisfies it and it remains valid under iterations.

Assumption 3 of the contour functional follows from the estimates of the partition function $\Xi_{W}^{(s)}(\gamma \mid \beta, \delta)$ in Theorem $\mathrm{B}$ and from the fact that for any smooth contour $\gamma_{1}=\left(b_{1}, p h\right)$ and contour $\gamma \in \operatorname{Sm}\left(\gamma_{1}\right)$ we have

$$
\left|\Delta^{(c r)}(\gamma \mid F)\right|+\left|\sum_{s} \Delta\left(O_{s}(\gamma) \mid F\right)\right| \leqq \text { v.s.n. }\left|b_{1}\right| .
$$

Having $F^{(f)}, F^{(3,3)}$ we find $\delta$ using the equation $a^{(3,3)}-a^{(f)}=2 \beta \delta$.

Peierls's method in principle does not give any possibility to approach a critical point. However, it is apparently possible to estimate the number of values of $\delta$ where the set of limit Gibbs states is discontinuous as a function of $\beta$. 
Acknowledgements. We express our sincere gratitude to V. Pokrovsky and G. Uimin for valuable discussions. Special thanks to A. Mazel who very carefully read the manuscript and gave us very many useful remarks which led to the substantial improvement of the text.

\section{References}

1. Domb, C.: On the theory of cooperative phenomena in crystals. Adv. Phys. 9, No. 34, 149 (1960)

2. Elliott, R.J.: Phenomenological discussion of magnetic ordering in the heavy-earth metals. Phys. Rev. 124, 346 (1961)

3. Bak, P., von Boehm, J.: Ising model with soliton, phasons and the "Devil's Staircase". Phys. Rev. B 21, 5297 (1980)

4. Fisher, M.E., Selke, W.: Low temperature analyses of the axial next-nearest neighbour Ising model near its multiphase point. Phil. Trans. Royal Soc. 302, 1 (1981)

5. Pirogov, S.A., Sinai, Ya.G.: Phase transitions of the first order for small perturbations of the Ising model. Funkt. Anal. Prilozh. 8, 25 (1974)

6. Sinai, Ya.G.: Theory of phase transitions. Rigorous results. Budapest: Acad. Kiado 1982

7. Zahradnik, M.: An alternate version of Pirogov-Sinai theory. Commun. Math. Phys. 94, 559581 (1984)

Communicated by J. Fröhlich

Received July 23, 1984 